Mitteilungen aus dem pharmazeutisch-chemischen Institut der Universitat Marburg.

Von Ernst Schmidt.

\title{
250. Ueber das Ephedrin und verwandte Verbindungen ${ }^{1}$ ).
}

Versuche von Dr. A ugust Eberhard, Apotheker.

\section{Ueber das Verhalten des Methylephedrin.Methylhydroxyds bel der Destillation seiner wăsserigen Lõsung.}

Der Zweck dieser Untersuchung war, wio bereits in der vorstehenden Arbeit (249) dargelegt wurde, die Ermittolung der stickstoffhaltigen Nebenprodukte, welche neben Trimethylamin und stickstofffreien Verbindungen bei der Destillation der wässerigen Lösung des Methylephedrin-Methylhydroxyds: $\mathrm{C}_{10} \mathrm{H}_{14}\left(\mathrm{CH}_{3}\right) \mathrm{ON}-\mathrm{CH}_{3} . \mathrm{OH}$, auftreten.

\section{Methylephedrin-Methyljodid: $\mathrm{C}_{10} \mathrm{H}_{14}\left(\mathrm{CH}_{3}\right) \mathrm{ON} . \mathrm{CH}_{3} \mathrm{~J}$.}

Die Darstellung des als Ausgangsmaterial für die Gewinnung des Methylephedrin-Methylhydroxyds verwendeten MethylephedrinMethyljodids erfolgte mit kleinen Modifikationen nach den Angaben von E. R. Mille $\mathrm{r}^{2}$ ) und H. $\mathrm{Emde} \mathrm{e}^{8}$. Das aus Ephedrinhydrochlorid-M e r c k dargestellte freie Ephedrin wurde zu diesem Zwecke in der 2-3 fachen Menge Methylalkohol gelöst, diese Lösung alsdann unter Abkühlung allmählich mit Jodmethyl im Ueberschuß versetzt und das Gemisch hierauf, nachdem die bereits bei gewöhnlicher Temperatur eintretende Reaktion beendet war, noch so lange am Rückflußkühler auf dem Wasserbade erhitzt, bis die Flüssigkeit neutrale Reaktion zeigte. Das im Ueberschuß angewendete Jodmethyl wurde alsdann im Verein mit dem Methylalkohol abdestilliert, wobei ein rotgefärbtes, beim Abkühlen krystallinisch erstarrendes Liquidum zurückblieb. Zur Entfernung des in diesem Reaktionsprodukt neben Methylephedrin-Methyljodid enthaltenen Methylephedrinhydrojodids

1) Inauguraldissertation, Marburg 1914.

2) Dieses Archiv 1902, 490.

8) Ibidem 1906, 252. 
wurde dasselbe in Wasser gelöst, die erzielte Lösung mit Barytwasser bis zur stark alkalischen Reaktion versetzt und das Gemisch hierauf wiederholt mit Aether ausgeschüttelt. Der nach dem Abdestillieren des Aethers verbleibende, aus Methylephedrin bestehende Rückstand wurde alsdann von neuem der Methylierung in obiger Weise unterworfen.

Aus der mit Aether ausgeschüttelten Lösung schied sich beim :Stehen bereits ein Teil des Methylephedrin-Methyljodids in würfelförmigen oder 'rechteckigen Krystallen aus. Der Rest wurde nach quantitativer Ausfällung des Baryumhydroxyds mit verdünnter Schwefelsäure durch Eindampfen gewonnen. Die weitere Reinigung erfolgte durch Umkrystallisieren aus siedendem Alkohol. Die auf diese Weise erhaltenen Krystalle schmolzen bei 2040. E. Mer $\mathrm{ck}^{1}$ ) und E. R. Miller (1. c.) fanden den Schmelzpunkt des Methylephedrin-Methyljodids bei $203^{\circ}$, H. Emde (1. c.) bei $199^{\circ}$.

\section{Methylephedrin-Methylchlorid: $\mathrm{C}_{10} \mathrm{H}_{14}\left(\mathrm{CH}_{3}\right) \mathrm{ON} \cdot \mathrm{CH}_{3} \mathrm{Cl}+\mathrm{H}_{2} \mathrm{O}$.}

Diese Verbindung wurde durch Umsetzen der wässerigen Lösung des Methylephedrin-Methyljodids mit frisch gefälltem Chlorsilber dargestellt. Nach dem Eindampfen der hierbei erzielten Lösung auf ein kleines Volum erstarrte im Exsikkator die ganze Flüssigkeit zu einer strahlig-krystallinischen Masse. Durch Umkrystallisieren aus wenig Wasser ließ sich jedoch dieses Produkt in große, durchsichtige, rechteckige Tafeln, welche bei $230^{\circ}$ schmolzen, überführen. Dieses Chlorid ist in Wasser und in Alkohol wesentlich leichter löslich als: das entsprechende Jodid. Im Exsikkator verliert dieses Chlorid bereits einen Teil seines Krystallwassers. Gewicht.

$0,2094 \mathrm{~g}$ lufttrockener Substanz verloren bei $100^{\circ} 0,0147 \mathrm{~g}$ an

Gefunden: Berechnet für $\mathrm{C}_{10} \mathrm{H}_{14}\left(\mathrm{CH}_{3}\right) \mathrm{ON}-\mathrm{CH}_{3} \mathrm{Cl}+\mathrm{H}_{2} \mathrm{O}$ :

\section{$\mathrm{H}_{2} \mathrm{O} \quad 7,02 \quad 7,27$}

$0,1223,1947 \mathrm{~g}$ der bei $100^{\circ}$ getrockneten Verbindung lieferten

Gefunden: Berechnet für $\mathrm{C}_{10} \mathrm{H}_{14}\left(\mathrm{CH}_{3}\right) \mathrm{ON}-\mathrm{CH}_{3} \mathrm{Cl}$ :

Cl 15,63

15,44

Die spezifische Drehung der wasserfreien Verbindung ergab sich als:

$$
[\alpha]_{D}^{10^{0}}=-30,54^{\circ} \text {. }
$$

$\left(1,0665 \mathrm{~g}\right.$ in $20,3468 \mathrm{ccm}$ Wasser gelöst; $1=2 \mathrm{dom} ; \mathrm{t}=15^{\circ}$ $\alpha=-3,202^{\circ}$.

1) Merck's Berichte 1883. 
Methylephedrin-Mathylhydroxyd: $\mathrm{C}_{10} \mathrm{H}_{14}\left(\mathrm{CH}_{3}\right) \mathrm{ON}$. $\mathrm{CH}_{3} . \mathrm{OH}$.

Dn das Methylephedrin-Methylhydroxyd kaum im roinon, krystallisierten Zustande zu erhalten ist, so wurden für die Spaltungsversuche clirckt die Jösungen verwendet, welche durch vorsichtigen Zusatz von frisch bereitetem, feuchtem Silberoxyd auf eine wässerige Lösung des Methylephedrin-Methyljodids erhalten wurden. Bei der Aufbowahrung einer derartigen Lösung schieden sich feine, weiße, bei $73^{\circ}$ schmelzende Nadeln wis, welche sich durch einen Vergleich mit der bei der Methylierung des Ephedrins als Nebenprodukt erhaltenen Verbindung als $M$ ethy.de phedrin erwiesen. Es erleidet somit das MethylephedrinMethylhydroxyd schon bei gewöhnlicher Temperatur allmählich eine Abspaltung von $\mathrm{CH}_{3} . \mathrm{OH}$.

Die Destillation der frisch hereiteten wässerigen Lösung des Methylephedrin-Methylhydroxyds erfolgte zumeist direkt auf der Asbestpappe in einer mit Kühler versehenen Retorte. Besonders gegen Ende der Destillation gingen hierbei beträchtlichere Mengen von Oeltröpfchen der stickstofffreien Spaltungsprodukte über. Der Retorteninhalt wurde daher bis nahe zur Trockne abdestilliert, der Rückstand alsdann mit $10 \mathrm{ccm}$ Wasser aufgenommen und die Destillation hierauf von neuem in dieser Weise ausgeführt. Obschon diese Destillationen so oft wiederholt wurden, als noch ein durch Oeltröpfchen getrübtes Destillat resultierte, bezüglich bis in der Retorte nur noch ein geringfügiger Rückstand verblieb, war doch die Ausbeute an stickstofffreiem Rohprodukt verhältnismäBig nur gering und sehr schwankend. Die aus je $20 \mathrm{~g}$ Methylephedrin-Methyljodid dargestellte quaternäre Base lieferte bei der Destillation ihrer wässerigen Lösung, als das Destillat wiederholt mit Aether ausgeschüttelt und letzterer mit $\mathrm{s} \mathrm{c} \mathrm{h} \mathrm{w} \mathrm{a} \mathrm{c} \mathrm{h}$ salzsäurehaltigem Wasser behandelt wurde, 5,2-6,5 $\mathrm{g}$ eines öligen, bei der Aufbewahrung auffallenderweise allmählich krystallinisch erstarrenden Produktes. Beim starken Abpressen desselben zwischen Fließpapier verblieben rein weiße, nadelförmige, bei $7^{\circ}$ schmelzende Krystalle. Durch Umkrystallisieren aus

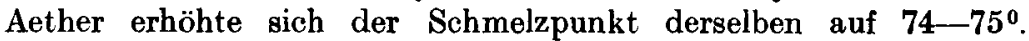
Die weitere Untersuchung lehrte, daß dieses Produkt aus Meth y le phedrin bestand.

$0,1952 \mathrm{~g}$ lieferten $0,5225 \mathrm{~g} \mathrm{CO}_{2}$ und $0,1635 \mathrm{~g} \mathrm{H}_{2} \mathrm{O}$.

Gefunden: Berechnet für $\mathrm{C}_{10} \mathrm{H}_{14}\left(\mathrm{CH}_{3}\right) \mathrm{ON}$ :

C 73,02

73,67

H $\quad 9,37$

9,56 
Methylephedrin: $\mathrm{C}_{10} \mathrm{H}_{14}\left(\mathrm{CH}_{3}\right) \mathrm{ON}$.

Das Methylephedrin zeichnet sich durch große Flüchtigkeit aus. Schon beim Aufbewahren im Exsikkator tritt eine ständige Gewichtsabnahme ein. In reinem und in schwach salzsäurehaltigem Wasser ist das Methylephedrin schwer löslich. In mäßig verdünnter Salzsäure ist die alkalisch reagierende Verbindung dagegen leicht löslich. Beim Verdunsten letzterer Lösung verbleibt ein in Wasser und Alkohol sehr leicht lösliches Hydrochlorid als eine weiße, krystallinische, bei $182-186^{\circ}$ schmelzende Masse.

Das Drehungsvermögen des Methylephedrins ergab sich als:

$$
[\alpha]_{\mathrm{D}}^{1 \mathrm{D}^{0}}=-18,96^{\circ} \text {. }
$$

$\left(0,8203 \mathrm{~g}\right.$ gelöst in $20,3232 \mathrm{ccm}$ Wasser; $t=15^{0} ; \mathrm{l}=2 \mathrm{dcm}$; $\alpha=-1,55^{\circ}$.)

Das Gold d o p pels a l z scheidet sich aus heißem Wasser in charakteristischen, starkglänzenden Blättchen aus, die bei $129^{\circ}$ schmelzen.

$0,3161 \mathrm{~g}$ enthielten $0,1198 \mathrm{~g} \mathrm{Au}$.

Gefunden: Berechnet für $\mathrm{C}_{10} \mathrm{H}_{14}\left(\mathrm{CH}_{3}\right) \mathrm{ON}, \mathrm{HCl}+\mathrm{AuCl}_{3}$ : Au 37,90 37,95

E. R. Mille r gibt den Schmelzpunkt des Methylephedrinaurats zu $121-123^{\circ}$, H. E m de zu $126^{\circ}$ an.

Das Platind oppels a l z resultierte nach wiederholtem Umkrystallisieren in ziemlich leicht löslichen, kleinen, rotgelben, bisweilen zu Drusen vereinigten Nadeln. Wasserfrei schmolz dieses Platinat bei $181^{\circ}$ und zersetzte sich unter Aufschäumen bei $184^{\circ}$.

$0,6856 \mathrm{~g}$ verloren bei $100^{\circ} 0,0314 \mathrm{~g}$ an Gewicht.

Gefunden: Berechnet für $\left[\mathrm{C}_{10} \mathrm{H}_{14}\left(\mathrm{CH}_{3}\right) \mathrm{ON}, \mathrm{HCl}_{2} \mathrm{PtCl}_{4}+2 \mathrm{H}_{2} \mathrm{O}\right.$ : $\mathrm{H}_{2} \mathrm{O} \quad 4,58 \quad 4,48$

$0,6542 \mathrm{~g}$ des getrockneten Platinats enthielten 0,173 $\mathrm{g} \mathrm{Pt}$.

Gefunden: $\quad$ Berechnet für $\left[\mathrm{C}_{10} \mathrm{H}_{1 \triangleleft}\left(\mathrm{CH}_{3}\right) \mathrm{ON}, \mathrm{HCl}_{2} \mathrm{PtCl}_{4}\right.$ :

Pt 25,23 25,39

Zur weiteren Identifizierung der als Spaltungsprodukt des Methylephedrin-Methylhydroxyds auftretenden Base diente das als Nebenprodukt der Methylierung des Ephedrins auftretende MethylEphedrin. Durch Umkrystallisieren aus Alkohol, bzw. aus Aether resultierte dasselbe in kurzen Nadeln oder in rechteckigen Täfelchen, welche bei $78^{\circ}$ schmolzen. Das sehr leicht lösliche Hydrochlorid schmolz bei $186^{\circ}$, das in glänzenden, goldgelben Blättchen krystallisierende Aurat (Goldgehalt $37,88 \%$ ) bei $129^{\circ}$. 


\begin{tabular}{|c|c|c|}
\hline & $\begin{array}{l}\text { Methylephedrin } \\
\text { aus Ephedrin }\end{array}$ & $\begin{array}{l}\text { Methylephedrinaus Methyl- } \\
\text { ephedrin-Methylhydroxyd }\end{array}$ \\
\hline Freie Base ... & $\begin{array}{c}\text { Nadeln oder Blättchen } \\
\text { Schmolzpunkt } 78^{\circ}\end{array}$ & $\begin{array}{c}\text { Nadeln } \\
\text { Schmelzpunkt } 74-75^{\circ}\end{array}$ \\
\hline Hydrochlorid. & $\begin{array}{c}\text { Leichtlösliche Täfelchen } \\
\text { oder krystallinische Massen } \\
\text { Schmelzpunkt } 186^{\circ}\end{array}$ & $\begin{array}{c}\text { Leichtlösliche } \\
\text { krystallinische Massen } \\
\text { Schmelzpunkt } 182--186^{\circ}\end{array}$ \\
\hline Aurat & $\begin{array}{l}\text { Goldgelbe, glänzende } \\
\text { Blättchen } \\
\text { Schmelzpunkt } 129^{\circ}\end{array}$ & $\begin{array}{l}\text { Goldgelbe, glänzende } \\
\text { Blättchen } \\
\text { Schmelzpunkt } 129^{\circ}\end{array}$ \\
\hline
\end{tabular}

Beim Eindampfen des Filtrats, welches bei der als Platinsulfid ausgeführten Platinbestimmung des als Spaltungsprodukt erhaltenen Methylephedrinplatinats gewonnen wurde, resultierten auffallenderweise farblose, bei $216^{0}$ schmelzende Krystalle, die wesentlich schwerer löslich waren, als die des Methylhydrochlorids. Dieselben stimmten im Schmelzpunkt und in den Löslichkeitsverhältnissen mit Ephedrinhydrochlorid überein. Es schien daher unter diesen Versuchsbedingungen die Abspaltung einer Methylgruppe eingetreten zu sein. Zur weiteren Prüfung dieses Verhaltens wurde reines, scharf bei $129^{\circ}$ schmelzendes Methylephedrinaurat durch Schwefelwasserstoff zerlegt und das Filtrat wiederholt mit Salzsäure auf dem Wasserbade eingedampft. Das hierbei gewonnene Hydrochlorid schmolz unscharf zwischen 184 und $200^{\circ}$. Es schien somit ein Gemisch von Ephedrin- und Methylephedrinhydrochlorid vorzuliegen. Zur Trennung dieser Hydrochloride wurden dieselben in möglichst wenig absolutem Alkohol gelöst und diese Lösung heiß mit siedendem Aceton versetzt. Beim längeren Stehen dieser Lösung schieden sich dann farblose, bei $210-211^{\circ}$ schmelzende Nadeln aus, die in ihrem Verhalten durchaus mit Ephedrinhydroehlorid übereinstimmten.

Beim Ueberschichten des etwas eingeengten Filtrats mit Aether schieden sich dann weiter kleine, zwischen 160 und $180^{\circ}$ schmelzende Nadeln aus, die im wesentlichen aus Methylephedrinhydrochlorid bestanden.

\section{Ephedrin.}

Die bei der Destillation der wässerigen Lösung des Methylephedrin-Methylhydroxyds gebildeten stickstofffreien Spaltungsprodukte waren infolge ihrer öligen Beschaffenheit beim Ab- 
pressen des Rohmethylephedrins (s. S. 64) vollständig von dem hierzu verwendeten Fließpapier aufgenommen worden. $\mathrm{Zu}$ ihrer Wiedergewinnung wurde dasselbe sorgfältig mit Aether extrahiert und die hierdurch erhaltene Lösung dann zur Entfernung der noch beigemengten stickstoffhaltigen Produkte mit Salzsäure ausgeschüttelt. Beim Verdunsten dieser salzsauren Lösung resultierten nadelförmige, bei $216^{\circ}$ schmelzende Krystalle, welche aus Ephedrinh ydroch lorid bestanden.

$0,2154 \mathrm{~g}$ lieferten $0,1533 \mathrm{~g} \mathrm{AgCl}$.

Gefunden:

Cl $\quad 17,59$
Berechnet für $\mathrm{C}_{10} \mathrm{H}_{15} \mathrm{ON}, \mathrm{HCl}$ : 17,57

Das entsprechende Ciolddoppelsalz enthielt $39,05 \%$ Au; berechnet für $\mathrm{C}_{10} \mathrm{H}_{15} \mathrm{ON}, \mathrm{HCl}+\mathrm{AuCl}_{3} 39,0 \%$. Die Mutterlaugen des Ephedrinhydrochlorids enthielten noch etwas $\mathrm{Methylephe-}$ d rin h y d roc hlorid.

Das Verbleiben eines beträchtlichen Teils des als Spaltungsprodukt des Methylephedrin-Methylhydroxyds gebildeten Methylephedrins und Ephedrins in dem ätherischen Auszuge dürfte larauf zurückzuführen sein, daß zum Ausschütteln desselben nur sehr schwach salzsaures Wasser, und zwar anscheinend in einer zur Bindung jener Basen unzureichenden Menge, verwendet worden war. Immerhin enthielt auch der schwach salzsaure Auszug jener Aetherlösung, wie dessen weitere Prüfung ergab, neben Trimethylamin auch noch reichliche Mengen von Methylephedrin und $b$ e sonders von Ephedrin. Zur Identifizierung dieser Produkte wurde dieser salzsäurehaltige Auszug zunächst auf ein sehr kleines Volum eingedampft und diese Lösung hierauf der Krystallisation überlassen. Hierbei gelangten warzenförmige, aus kleinen Nadeln bestehende Gebilde zur Abscheidung. Von diesen Massen löste sich der größte Teil ziemlich leicht in kaltem Wasser, während ein kleiner, aus sandähnlichen Körnchen bestehender Teil sich darin nicht auflöste.

Dieser schwer lösliche Anteil lieferte beim Umkrystallisieren aus wenig siedendem Wasser durchsichtige, tafelförmige Krystalle, die bei $133-136^{\circ}$ schmolzen.

$0,0796 \mathrm{~g}$ lieferten $0,0343 \mathrm{~g} \mathrm{AgCl}$, entsprechend 10,65\% Cl.

In seinen physikalischen Eigenschaften und in dem Chlorgehalt stimmt dieses Chlorid mit dem im nachstehenden (s. S. 75) beschriebenen Hydrochlorid des Ephedrin-Phenylp r o p y le $n$ o x y d s: $\mathrm{C}_{19} \mathrm{H}_{25} \mathrm{NO}_{2}, \mathrm{HCl}$, überein, dessen Chlorgehalt $10,56 \%$ beträgt. 
Da die in Wasser leichter löslichen Anteile nach ihrem Verhalten aus einem Gemisch verschiedener Hydrochloride bestanden, wurde die Lösung derselben zur Trockne verdampft und der Rückstand zur Trennung der Einzelbestandteile mit siedendem Aceton am Rückflußkühler behandelt. Beim Erkalten dieses Auszuges schied sich eine kleine Menge von nadelförmigen, bei 163- $177^{\circ}$ schmelzenden Krystallen mit einem Chlorgehalt von $16,79 \%$ aus. Dieses Produkt dürfte im wesentlichen aus dem in Aceton leicht löslichen Meth glephedrin hydrochlorid, welches $16,45 \% \mathrm{Cl}$ enthält, bestanden haben. Auch in der Acetonmutterlauge schien, nach den Eigenschaften und dem Goldgehalt. der daraus dargestellten Aurate zu urteilen, neben Trimethylamin (für das Aurat gefunden: 48,83\% Au, berechnet 49,4\%), Methylephedrin (für das Aurat gefunden: $37,43 \% \mathrm{Au}$, berechnet $37,95 \%$ ) noch enthalten zu sein.

Aus der Mutterlauge des Methylephedrinaurats schieden sich als zweite Krystallisation seidenglänzende, bei $148^{\circ}$ schmelzende Nadeln aus, die sich boim Umkrystallisieren in stark glänzende, rötlichgelbe Blättchen vom Schmelzpunkt $154-155^{\circ}$ verwandelten.

1. $0,2326 \mathrm{~g}$ enthielten $0,0879 \mathrm{~g} \mathrm{Au}=37,79 \%$.

2. $0,3242 \mathrm{~g} \quad " \quad 0,1222 \mathrm{~g} \mathrm{Au}=37,69 \%$.

Das entsprechende Platindoppelsalz krystallisierte in blaßroten, bei $218-219^{\circ}$ schmelzenden Blättchen.

\section{$0,1573 \mathrm{~g}$ enthielten $0,0406 \mathrm{~g} \mathrm{Pt}=25,8 \%$.}

Obschon die ermittelten analytischen Daten mit den von den bezüglichen Doppelsalzen des Methylephedrins verlangten Werten in Einklang stehen, so ist jedoch in den Eigenschaften derselben keine Uebereinstimmung (Methylephedrinaurat schmilzt bei 1290, Methylephedrinplatinat bei $\left.168-170^{\circ}\right)$ zu konstatieren. Welcher Natur die betreffende Base ist, mag zunächst dahingestellt bleiben.

Der bei der Behandlung mit heißem Aceton ungelöst gebliebene Teil des obigen Hydrochlorids wurde zur Identifizierung in wenig heißem Methylalkohol gelöst und diese Lösung dann mit heißem Aceton versetzt. Beim Erkalten schieden sich farblose, bei $210,5^{\circ}$ schmelzende Nadeln von Ephedrinhydroc h l.orid ab.

$0,2732 \mathrm{~g}$ ergaben $0,1969 \mathrm{~g} \mathrm{AgCl}$.

Gefunden: Berechnet für $\mathrm{C}_{10} \mathrm{H}_{18} \mathrm{ON}, \mathrm{HCl}$ :

Um das bei den im vorstehenden beschriebenen Spaltungsversuchen des Methylephedrin-Methylhydroxyds isolierte E phe- 
d $\mathbf{r}$ in weiter als solches zu kennzeichnen, wurden dieselben in der angegebenen Weise wiederholt. Die hierbei gewonnenen Destillate wurden jedoch vor dem Ausschütteln der stickstofffreien Spaltungsprodukte mit Aether zur vollständigen Bindung der gleichzeitig gebildeten basischen Produkte, stark mit Salzsäure angesäuert. Unter diesen Bedingungen zeigte der nach dem Abdestillieren des Aethers verbleibende, angenehm nach Dill und Estragon riechende ölige Rückstand auch bei längerer Aufbewahrung keine Neigung zur Krystallisation.

Beim Eindampfen der stark sauren wässerigen Flüssigkeit schieden sich beträchtliche Mengen von farblosen, nadel- und tafelförmigen Krystallen von Ephedrinhydrochlorid aus. Dieselben schmolzen bei $213,5^{\circ}$. In kaltem Wasser lösten sich lieselben leicht vollständig auf. Das bei den früheren Spaltungs versuchen isolierte schwer lösliche, bei $133-136^{\circ}$ schmelzende Hydrochlorid des Phenylpropylenoxyd-Ephedrins: $\mathrm{C}_{\mathbf{1 0}} \mathrm{H}_{\mathbf{2 5}} \mathrm{NO}_{\mathbf{2}}, \mathrm{HCl}$, konnte hierbei nicht beobachtet werden.

\section{$0,2638 \mathrm{~g}$ lieferten $0,188 \mathrm{~g} \mathrm{AgCl}$.}

Gefunden:
Cl 17,61

Das Drehungsvermögen dieses Hydrochlorids steht mit den Beobachtungen, welche früher bei dem Ephedrinhydrochlorid gemacht wurden, im Einklang:

$$
\text { a) }[a]_{D}^{15^{\circ}}=-34,15^{\circ} \text {. }
$$

$\left(0,9338 \mathrm{~g}\right.$ gelöst in $20,342 \mathrm{ccm}$ Wasser; $a=-3,138^{\circ} ; 1=2 \mathrm{dcm}$.)

b) $[a]_{D}^{150}=-34,48^{\circ}$.

$\left(0,9954 \mathrm{~g}\right.$ gelöst in $20,342 \mathrm{ccm}$ Wasser; $a=-3,374^{\circ} ; 1=2 \mathrm{dcm}$.)

c) $[\alpha]_{\mathrm{D}}^{15^{\circ}}=-35,8^{\circ}$.

$\left(1,0079 \mathrm{~g}\right.$ gelöst in $20,342 \mathrm{ccm}$ Wasser; $\alpha=--3,55^{\circ} ; 1=2 \mathrm{dcm}$.)

Das aus diesem Hydrochlorid dargestellte Golddoppelsalz schied sich, wie dies bei dem Ephedrin bisweilen auch der Fall ist, zunächst ölig ab, jedoch ließ es sich ohne Schwierigkeiten’in nadelförmige, bei $130-131^{\circ}$ schmelzende Krystalle überführen.

$$
\begin{aligned}
& \text { 0,5311 g enthielten } 0,2083 \mathrm{~g} \text { Au. } \\
& \text { Gefunden: } \\
& \begin{array}{c}
\mathrm{Au} 39,22 \\
\mathbf{3}
\end{array}
\end{aligned}
$$

Die Mutterlaugen des Ephedrinhydrochlorids enthielten auch hier die Hydrochloride des Methylephedrins und Trimeth y a mins. Ein bei $154-155^{\circ}$ schmelzendes Golddoppelsalz (s. S. 68) konnte dagegen nicht daraus gewonnen werrlru. 
Als stickstoffhaltige Spaltungsprodukte traten somit bei der Destillation der wässerigen Lösung des Methylephedrin-Methylhydroxyds auf: Trimethy la min, Methylephedrin und Ephedrin, sowie in geringer Menge durch Vereinigung (ler betreffenden Komponenten gebildet, $\mathrm{E} p \mathrm{~h}$ ed $\mathrm{r}$ in- $\mathrm{Ph}$ en y lpropylenoxyd.

\section{Spaltung des Mothylophedrin-Mothyljodids durch Natriumamalgam.}

Die Einwirkung des Natriumamalgams auf MethylephedrinMethyljodid gelangte entsprechend den Angaben von $\mathrm{H}$. E m d e ") über die Spaltung quaternärer Ammoniumverbindungen zur Ausführung. Vorversuche lehrten zunächst, đaß auch das Methylephedrin-Methyljodid unter dem Einfluß des Natriumamalgams eine Zersetzung erfährt, und daß dabei ähnliche Produkte auftreten, wie bei der Destillation der wässerigen Lösung des Methylephedrin-Methylhydroxyds. Stickstofffreie Verbindungen konnten allerdings hierbei nur in geringer Menge isoliert werden, dagegen wurde von stickstoffhaltigen Produkten das Auftreten von T rimethylamin, Methylephedrin, Ephedrin und vor allem von Ephedrin - Phenylpropylenoxyd konstatiert. Das Mengenverhältnis in welchem diese basischen Verbindungen, besonders die letztere, unter diesen Versuchshedingungen auftreten, hängt in erster Linie ab von der Konzentration der zur Anwendung gelangenden wässerigen Lösung cles Methylephedrin-Methyljodids, weniger von der Menge des in Reaktion versetzten Natriumamalgams ron $5 \%$.

Das Natriumamalgam wurde in einer Menge von $320 \mathrm{~g}$ und $450 \mathrm{~g}$ auf je $20 \mathrm{~g}$ Methylephedrin-Methyljodid angewendet. Die beste Ausbeute $(6 \mathrm{~g})$ an dem Hydrochlorid des Ephedrin-Phenylpropylenoxyds wurde bei Anwendung einer im Verhältnis von 1:5 heiß bereiteten wässerigen Lösung und $320 \mathrm{~g}$ Natriumamalgam von $5 \%$ auf $20 \mathrm{~g}$ Methylephedrin-Methyljodid erzielt.

\section{๙) Spaltung des Methylephedrin-Methyljodids (20 g) durch $320 \mathrm{~g}$ Natriumamalgam.}

$20 \mathrm{~g}$ fein zerriebenes Methylephedrin-Methyljodid wurden in $100 \mathrm{ccm}$ Wasser im siedenden Wasserbade am Rückflußkühler in einem Kolben gelöst und in diese heiße Lösung $320 \mathrm{~g}$ Natriumamalgam von $\mathbf{5 \%}$ unter häufigem Umschütteln in kleinen Portionen allmählich eingetragen. Schon nach dem Eintragen der ersten

1) Dieses Arehiv 1911, 110. 
Anteile des Natriumamalgams trat der Geruch nach Trimethylamin auf, neben welchem sich auch bald der bei der Destillation der wässerigen Lösung des Methylephedrin-Methylhydroxyds beobachtete, durch die stickstofffreien Spaltungsprodukte bedingte Dill- und Estragongeruch bemerkbar machte. Gleichzeitig erfolgte an der Oberflüche der Flüssigkeit die Abscheidung eines braungefärbten, öligen Liquidums, dessen Menge sich in dem Maße vermehrte, als der weitere Zusatz von Natriumamalgam erfolgte.

Nachdem alles Natriumamalgam eingetragen war und auch bei weiterem Erwärmen des Reaktionsproduktes keine Entwickelung von Wasserstoff mehr eintrat, wurde durch das noch warme Gemisch ein Luftstrom zur Entfernung des gebildeten Trimethylamins geleitet. Hierauf wurde die alkalische Flüssigkeit von dem abgeschiedenen Quecksilber abgegossen und wiederholt mit Aether ausgeschüttelt. Die vereinigten Aetherauszüge wurden alsdann, zur Trennung der stickstofffreien und der stickstoffhaltigen, basischen Spaltungsprodukte, wiederholt durch Schütteln mit stark salzsäurehaltigem Wasser extrahiert.

Nach dem Eindampfen dieser salzsäurehaltigen Auszüge schied sich zunächst eine geringe Menge bräunlich gefärbter, bei 83-840 schmelzender Krystalle aus. Dieselben wurden mit der zweiten, reichlioheren Krystallausscheidung vereinigt und aus heißem Wasser, unter Zusatz von etwas Tierkohle, umkrystallisiert. Auf diese Weise wurden farblose, in kaltem Wasser ziemlich schwer lösliche, tafelförmige Krystalle erhalten, welche bei $142^{\circ}$ schmolzen. Dieselben bestanden nach ihren Eigenschaften und dem Chlorgehalt aus dem Hydrochlorid des EphedrinPhenylpropylenoxyds (s. unten), einer Base, welche in geringer Menge auch aus dem Destillat der wässerigen Methylephedrin-Methylhydroxydlösung isoliert werden konnte (s. S. 67). Die gleiche Verbindung konnte, wenn auch zunächst in weniger reiner Gestalt, auch noch aus der weiteren Mutterlauge gewonnen worden.

Die letzten Mutterlaugen zeigten wenig Neigung zur Krystallisation; sie wurden daher mit den beim Umkrystallisieren des Ephedrin-Phenylpropylenoxydhydrochlorids erhaltenen vereinigt, mit Soda alkalisch gemacht und dann mit Aether ausgeschüttelt. Dieses Aetherextrakt wurde hierauf von neuem in ein Hydrochlorid verwandelt und dessen Lösung der Krystallisation überlassen. Hierbei schied sich zunächst noch eine geringe Menge des schwer löslichen Hydrochlorids des E phedrin-P.h en y l - 
propylenoxyds aus. Die Mutterlauge lieferte alsdann Krystallisationen, die nach dem Schmelzpunkt und dem sonstigen Verhalten aus einem Gemisch von Ephedrin- und Methylephedrinhydrochlorid bestanden. $\mathrm{Zu}$ deren Trennung wurde dies Gemisch in wenig heißem Alkohol gelöst und diese Lösung mit heißem Aceton versetzt. Beim Erkalten und beim ruhigen Stehen in verschlossenem Gefäße schieden sich farblose, bei $210-211,5^{\circ}$ schmelzende Nadeln von Ephedrinhydrochlorid aus.

$0,2549 \mathrm{~g}$ lieferten $0,1815 \mathrm{~g} \mathrm{AgCl}$.
Gefunden :
Cl 17,64
Berechnet für $\mathrm{C}_{10} \mathrm{H}_{15} \mathrm{ON}, \mathrm{HCl}$ :
17,58

Aus der Mutterlauge des Ephedrinhydrochlorids konnten noch rechteckig ausgebildete, bei $184-185^{\circ}$ schmelzende Blättchen von Meth y lephedrin y d roc h lorid isoliert werden.

Als stickstoffhaltige Spaltungsprodukte waren somit unter obigen Versuchsbedingungen gebildet: Trimethylamin, Ephedrin, Methylephedrin und EphedrinPhenylpropylenoxyd, dessen Hydrochlorid bei Anwendung einer möglichst konzentrierten, mindestens $1: 5$ bereiteten wässerigen Lösung des Methylephedrin-Methyljodids das Hauptzersetzungsprodukt bildete, so daß es bei Anwendung von $20 \mathrm{~g}$ dieses Jodids leicht in einer Menge von $6 \mathrm{~g}$ isoliert werden konnte. Bei Behandlung der ätherischen Ausschüttelung des alkalisohen Reaktionsproduktes mit verdünnter Salzsäure schied sich unter diesen Bedingungen das Hydrochlorid des Ephedrin-Phenylpropylenoxyds direkt als lockere, weiße, krystallinische Flocken in solcher Menge aus, daß die ganze Flüssigkeit damit erfüllt war.

Die Menge der stickstoffreien Spaltungsprodukte, welche nach dem Abdestillieren der wiederholt mit verdünnter Salzsäure ausgeschüttelten Aetherauszüge des Reaktionsproduktes zurückblieb, war eine sehr geringe. Dieselben bildeten ein gelblich gefärbtes, öliges, nach Dill und Estragon riechendes Liquidum.

Der mit Aether ausgeschüttelte alkalische Rückstand der Einwirkung des Natriumamalgams auf Methylephedrin-Methyljodid enthielt noch einen kleinen Teil des angewendeten Jodids, welches sich der Reaktion entzogen hatte. Dasselbe konnte in blaßgelben, kompakten, bei $196,5^{\circ}$ schmelzenrlen Krystallen daraus isoliert werden.

$$
\begin{aligned}
& 0,1891 \mathrm{~g} \text { lieferten } 0,1389 \mathrm{~g} \text { AgJ. } \\
& \text { Gefunden: } \\
& \text { J 39,71 } \\
& \text { Berechnet für } \mathrm{C}_{10} \mathrm{H}_{1 \mathrm{~s}} \mathrm{ON}, \mathrm{CH}_{8} \mathrm{~J} \text { : } \\
& 39,56
\end{aligned}
$$


ß) Spaltung des Methylephedrin-Methyljodids (20 g) durch $450 \mathrm{~g}$ Natriumamalgam.

Die Einwirkung dieser größeren Menge Natriumamalgam von $5 \%$ gelangte auf die heiße, 1:5 bereitete wässerige Lösung des Methylephedrin-Methyljodids in gleicher Weise zur Ausführung, wie es im vorstehenden für die geringere Menge angegeben ist. Auch die weitere Verarbeitung des Reaktionsproduktes war die gleiche.

Aus der durch Ausschütteln mit verdünnter Salzsäure aus dem Aetherauszuge des Reaktionsproduktes gewonnenen Lösung wurden zunächst beträchtliche Mengen des schwer löslichen, bei $143^{\circ}$ schmelzenden Hydrochlorids des Ephedrin-Phenylpropy le nox y d s isoliert.

$0,1575 \mathrm{~g}$ lieferten 0,0656 $\mathrm{g} \mathrm{AgCl}$.

$\begin{array}{cc}\text { Gefunden: } & \text { Berechnet für } \mathrm{C}_{10} \mathrm{H}_{25} \mathrm{NO}_{2}, \mathrm{HCl} \text { : } \\ \mathrm{Cl} 10,30 & 10,56\end{array}$

Die Ausbeute an diesem Hydrochlorid war jedoch etwas geringer als bei Anwendung von $320 \mathrm{~g}$ Natriumamalgam. Aus der Mutterlauge dieses Hydrochlorids resultierten dann einige tafelförmige, bei $183^{\circ}$ schmelzende Krystalle von Methylephedrinhydrochlorid. Die letzten Mutterlaugen erstarrten schließlich zu einer krystallinischen Masse. Durch Lösen derselben in wenig heißem Alkohol und Zufügen von heißem Aceton gelang es, daraus nadelförmige, bei $212^{\circ}$ schmelzende Krystalle von $\mathrm{Ephedrinhydrochlorid} \mathrm{zur} \mathrm{Abscheidung}$ $\mathrm{zu}$ bringen.

$0,2016 \mathrm{~g}$ lieferten $0,1437 \mathrm{~g} \mathrm{AgCl}$.

Gefunden:
Cl 17,60

Das Drehungsvermögen dieses Hydrochlorids stimmte mit dem des naturellen Ephedrins überein. Es ergab sich zunächst als $[\alpha]_{D}^{15^{0}}=-34,31^{0}$.

$\left(0,9264 \mathrm{~g}\right.$ gelöst in $20,1183 \mathrm{ccm}$ Wasser; $1=2 \mathrm{dcm} ; \alpha=-3,16^{\circ}$.)

Nach nochmaliger Umkrystallisation wurde bei dem nun bei $215^{\circ}$ scbmelzenden Hydrochlorid gefunden:

$$
[\alpha]_{D}^{150}=-36,23^{0} .
$$

$\left(1,000 \mathrm{~g}\right.$ gelöst in 20,351 ccm Wasser; $\left.1=2 \mathrm{dcm} ; \alpha=-3,56^{\circ}.\right)$

Das aus diesem Hydrochlorid dargestellte Gold d o p p e l$\mathrm{salz}$ bildete nadelförmige, wie das Ephedrinaurat bei $131^{\circ}$ schmelzende Krystalle. 
$0,2372 \mathrm{~g}$ enthielten $0,0921 \mathrm{~g} \mathrm{Au}$.

Gefunden: $\quad$ Berechnet für $\mathrm{C}_{10} \mathrm{H}_{15} \mathrm{ON}, \mathrm{HCl}+\mathrm{AuCl}_{3}$ : Au $\quad 38,83$ 39,0

Das entsprechende P la t i n d o p pe $1 \mathrm{~s}$ a $1 \mathrm{z}$ bildete ziemlich leicht lösliche, rotgelbe, bei $183^{\circ}$ schmelzende, nadelförmige Krystalle. Ephedrinplatinchlorid schmilzt bei $183-184^{\circ}$.

$0,1519 \mathrm{~g}$ enthielten $0,0399 \mathrm{~g}$ Pt.

$\begin{array}{cc}\text { Gefunden : } & \text { Berechnet für } \underset{\mathbf{2 6 , 3 2}}{\left(\mathrm{C}_{10} \mathrm{H}_{13} \mathrm{ON}, \mathrm{HCl}\right)_{2} \mathrm{PtCl}_{4} \text { : }} \text { Pt 26,27 }\end{array}$

Die durch Reduktion des Methylephedrin-Methyljodids gebildete Base der Formel $\mathrm{C}_{10} \mathrm{H}_{15} \mathrm{ON}$ stimmt somit in allen ihren Eigenschaften mit dem naturellen $\mathrm{E} p \mathrm{~h}$ ed $\mathrm{r}$ in überein.

Die Alkohol-Aceton-Mutterlauge des Ephedrinhydrochlorids lieferte bei langsamer Verdunstung eine gelblich gefärbte, krystallinische Masse, welche bei $164-178^{\circ}$ schmolz. Beim Umkrystallisieren aus wenig heißem absoluten Alkohol resultierte etwa $1 / 3$ dieser Masse in farblosen, gut ausgebildeten, tafelförmigen Krystallen, welche bei $184-186^{\circ}$ schmolzen. Dieselben stimmten in der Krystallform, in dem Chlorgehalt und in dem Schmelzpunkt mit Methyle phedrinhydrochlorid überein.

$0,2211 \mathrm{~g}$ lieferten $0,148 \mathrm{~g} \mathrm{AgCl}$. Gefunden :
Cl 16,55
Berechnet tür $\mathrm{C}_{10} \mathrm{H}_{14}\left(\mathrm{CH}_{3}\right) \mathrm{ON}, \mathrm{HCl}$ : 16,45

Das aus diesem Hydrochlorid dargestellte G o I d d o p p e l s a lz bildete glänzende, blätterige, bei $125-127^{\circ}$ schmelzende Krystalle.

$0,2618 \mathrm{~g}$ enthielten 0,0997 $\mathrm{g} \mathrm{Au}$.

Gefunden: $\quad$ Berechnet für $\mathrm{C}_{10} \mathrm{H}_{14}\left(\mathrm{CH}_{3}\right) \mathrm{ON}, \mathrm{HCl}+\mathrm{AuCl}_{3}$ : Au 38,08 37,95

Die alkoholische Mutterlauge des Methylephedrinhydrochlorids lieferte bei weiterer langsamer Verdunstung nur noch wenige quadratische, bei $186-187^{\circ}$ schmelzende Krystalle dieser Verbindung. Der Rest der Mutterlauge trocknete allmählich zu einer krystallinischen Masse ein, welche anscheinend aus einem Gemisch von viel Methylephedrinhydrochlorid mit wenig Ephedrinhydrochlorid bestand. Die daraus dargestellten Golddoppelsalze schmolzen bei $118-119^{\circ}$, bei $119-121^{\circ}$ und bei $131^{\circ}$. Dieselben enthielten $38,3 \%, 37,92 \%$ und $38,46 \%$ Au. Das bei $131^{\circ}$ schmelzende Ephedrinaurat enthält $39,0 \% \mathrm{Au}$, das bei $129^{\circ}$ schmelzende Methylephedrinaurat 37,95\% Au. 
Die Mengen von E $\mathrm{ph}$ ed rin - und Meth y le phedrin h ydrochlorid, welche aus dem Einwirkungsprodukte von 450 g $5 \%$ igen Natriumamalgam auf 20 g Methylephedrin-Methyljodid isoliert werden konnten, waren beträchtlicher als bei der Anwen-

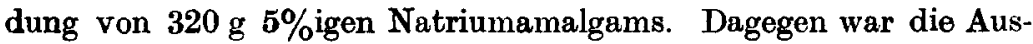
beute an stickstofffreien Produkten auch bei der Anwendung von $\mathbf{4 5 0} \mathrm{g}$ Natriumamalgam, ebenso wie bei der Anwendung von $320 \mathrm{~g}$ $5 \%$ igen Natriumamalgams, nur eine sehr geringe.

\section{Ephedrin-Phenylpropylenoxyd : $\mathrm{C}_{10} \mathrm{H}_{15} \mathrm{ON} \cdot \mathrm{C}_{9} \mathrm{H}_{10} \mathrm{O}$.}

$\mathrm{Hydrochlorid:} \mathrm{C}_{10} \mathrm{H}_{15} \mathrm{ON} \cdot \mathrm{C}_{9} \mathrm{H}_{10} \mathrm{O}, \mathrm{HCl}$. Das bei der Einwirkung von Natriumamalgam auf heiße wässerige Methylephedrin-Methyljodidlösung, je nach der Konzentration derselben, in kleinerer oder größerer Menge erhaltene Hydrochlorid des Ephedrin-Phenylpropylenoxyds bildete kompakte farblose, durchsichtige, rechteckige Tafeln, welche im reinen Zustande bei 146 bis $148^{\circ}$ stark zusammensinterten, jedoch erst bei $155-156^{\circ}$ unter schwachem Aufschäumen vollständig klar schmolzen. Bisweilen erfolgte nach dem vollständigen Schmelzen eine krystallinische Ausscheidung, die dann erst über $190^{\circ}$ schmolz. Es scheint somit das Hydrochlorid des Ephedrin-Phenylpropylenoxyds beim Schmelzen unter Umständen eine Spaltung zu erleiden.

In kaltem Wasser und in Alkohol war dieses Hydrochlorid schwer löslich.

1. $0,1575 \mathrm{~g}$ lieferten $0,0656 \mathrm{~g} \mathrm{AgCl}$.

2. $0,2335 \mathrm{~g} \quad, \quad 0,5746 \mathrm{~g} \mathrm{CO}_{2}$ und $0,1718 \mathrm{~g} \mathrm{H}_{2} \mathrm{O}$.

3. $0,1934 \mathrm{~g} \mathrm{,} \quad 7,1 \mathrm{ccm}$ Stickstoff bei $19,6^{\circ}$ und $756 \mathrm{~mm}$ Druck.

\begin{tabular}{ccccc} 
& \multicolumn{3}{c}{ Gefunden : } & Berechnet für \\
& 1. & 2. & 3. & $\mathrm{C}_{19} \mathrm{H}_{25} \mathrm{NO}_{2}, \mathrm{HCl}:$ \\
$\mathrm{Cl}$ & 10,30 & - & - & 10,56 \\
$\mathrm{C}$ & - & 67,11 & - & 67,24 \\
$\mathrm{H}$ & - & 8,13 & - & 7,97 \\
$\mathrm{~N}$ & - & - & 4,12 & 4,17
\end{tabular}

Die Bestimmung seines spezifischen Drehungsvermögens ergab: a) $[\alpha]_{D}^{15^{0}}=+14,74^{0}$.

$\left(0,5598 \mathrm{~g}\right.$ in 20,3474 $\mathrm{ccm}$ Alkohol gelöst; $\left.\alpha=\uparrow \cdot 0,84^{0} ; 1=2 \mathrm{dcm}.\right)$

b) $[a]_{\mathrm{D}}^{15^{0}}=+14,93^{0}$.

$\left(0,3047 \mathrm{~g}\right.$ in $20,3474 \mathrm{ccm}$ Alkohol gelöst; $\alpha=+0,45^{\circ} ; 1=2 \mathrm{dom}$.)

Durch orneute Behandlung der heißen wässerigen Lösung dieses Hydrochlorids mit Natriumamalgam von $5 \% \quad(70 \mathrm{~g}$ 
A. Eberhard: Ephedrin und verwandte Verbindungen.

auf $1 \mathrm{~g}$ Hydrochlorid) erlitt diese Verbindung keine Veränderung. Dieselbe konnte daher unverändert wieder aus dem Reaktionsprodukte isoliert werden.

Natriumkarbonat scheidet aus der wässerigen Lösung dieses Hydrochlorids die freie Base aus, welche dann durch Ausschütteln der Mischung mit Aether leicht isoliert werden kann.

Frei e $\mathrm{B}$ a s e. Die diesem Hydrochlorid entsprechende freie Base scheidet sich beim langsamen Verdunsten ihrer ätherischen Lösung in farblosen, tafelförmigen, Parallelogrammform zeigenden Krystallen aus. Diese Krystalle erweichen bei $123^{\circ}$ und schmelzen bei $125^{\circ}$.

$0,200 \mathrm{~g}$ lieferten $0,657 \theta \mathrm{g} \mathrm{CO}_{2}$ und $0,1522 \mathrm{~g} \mathrm{H}_{2} \mathrm{O}$.

\begin{tabular}{|c|c|}
\hline Gefunden: & Berechnet für $\mathrm{C}_{19} \mathrm{H}_{25} \mathrm{NO}_{2}$ : \\
\hline C $\quad \mathbf{7 6 , 0 4}$ & 76,20 \\
\hline H $\quad 8,51$ & 8,42 \\
\hline
\end{tabular}

Die Bestimmung des spezifischen Drehungsvermögens ergab:

a) $[\alpha]_{D}^{150}=-3,59^{\circ}$.

$\left(0,4771 \mathrm{~g}\right.$ in $20,3474 \mathrm{ccm}$ Alkohol gelöst; $\alpha=-0,17^{\circ} ; 1=2 \mathrm{dcm}$.)

b) $[\alpha]_{D}^{15^{0}}=-3,63^{\circ}$.

$\left(0,9098 \mathrm{~g}\right.$ in $20,3474 \mathrm{ccm}$ Alkohol gelöst; $\alpha=-0,33^{\circ} ; 1=2 \mathrm{dcm}$.)

Gold d op pelsalz. Das aus obigem. Hydrochlorid dargestellte Aurat ist in Wasser schwer löslich. Leichter löst es sich in mäßig verdünntem Alkohol. Aus heißem, stark salzsäurehaltigem Wasser schied es sich in gelben, glänzenden, feinen Nadeln oder Blättchen aus, die bei $144-145^{\circ}$ schmolzen.

1. 0,3597 $\mathrm{g}$ enthielten $0,1095 \mathrm{~g}$ Au.

2. $0,2978 \mathrm{~g}, \quad 0,0915 \mathrm{~g}$ Au.

Gefunden :

1. 2.

Au $\quad 30,44 \quad 30,72$

Berechnet für $\mathrm{C}_{19} \mathrm{H}_{25} \mathrm{NO}_{2}, \mathrm{HCl}+\mathrm{AuCl}_{3}$ : 30,85

Platind op pelsalz. Das Platinat dieser Base ist in Wasser wesentlich leichter löslich als das Aurat. Dasselbe schied sich in warzenförmig angeordneten Nadeln aus, welche bei $176,5-177^{\circ}$ sehmolzen.

1. $0,2185 \mathrm{~g}$ des bei $100^{\circ}$ getrockneten Platinats enthielten $0,0431 \mathrm{~g} \mathrm{Pt}$.

2. $0,2640 \mathrm{~g}$ des bei $100^{\circ}$ getrockneten Platinats enthielten $0,0512 \mathrm{~g} \mathrm{Pt}$.

Gefunden:

1. 2.

Pt $\quad 19,72 \quad 19,40$
Berechnet für

$\left(\mathrm{C}_{10} \mathrm{H}_{20} \mathrm{NO}_{2}, \mathrm{HCl}\right)_{2} \mathrm{PtCl}_{4}$ :

19,35 
Jodmethylat. Schon die Fällbarkeit der fraglichen Base aus der wässerigen Lösung ihres Hydrochlorids durch Natriumkarbonat und die leichte Löslichkeit derselben in Aether wies darauf hin, daß dieselbe nicht den Charakter einer quaternären Verbindung besitzen und sich somit nicht vom Methylephedrin, sondern vermutlich vom Ephedrin ableiten konnte. Zur weiteren Prüfung dieser Annahme wurden 0,5 g der freien Base mit Jodmethyl im Ueberschuß zusammengebracht und dieses Gemisch dann in einem verschlossenen Gefäße längere Zeit bei gewöhnlicher Temperatur sich selbst überlassen. Hierbei schieden sich aus der anfänglich klaren Lösung allmählich Krystalle aus. Nach Verlauf von 14 Tagen wurde dann das im Ueberschuß angewendete Jodmethyl verdunstet und der Rückstand in heißem Wasser gelöst. Hierbei blieb eine geringe Menge desselben ungelöst, die sich bei näherer Prüfung als unverändert gebliebenes Ephedrin-Phenylpropylenoxyd herausstellte. Aus der erhaltenen wässerigen Lösung schieden sich dagegen kompakte, gelbliche, bei $183^{\circ}$ schmelzende Krystalle eines Jodids aus. Das hieraus dergestellte, wesentlich leichter lösliche Chlorid bildete flache, durchsichtige, bei $203^{\circ}$ schmelzende Nadeln.

$0,1181 \mathrm{~g}$ lieferten 0,0485 g AgCl.

$\begin{array}{cc}\text { Gefunden: } & \text { Berechnet für } \mathrm{C}_{10} \mathrm{H}_{23} \mathrm{NO}_{2} \cdot \mathrm{CH}_{3} \mathrm{Cl} \text { : } \\ \mathrm{CI} 10,18 & 10,14\end{array}$

Ein P latind o p pels a l z konnte in krystallisierter Form von diesem Chlorid nicht erhalten werden.

Die Mutterlaugen des Jodids und Chlorids wurden, im Verein mit der Lösung der in krystallisierter Form erhaltenen Verbindungen, durch vorsichtigen Zusatz von feuchtem Silberoxyd von Halogen befreit. Es resultierte hierbei eine stark alkalisch reagierende Flüssigkeit, welche beim wiederholten Ausschütteln mit Aether an diesen nur Spuren eines gelblichen Oeles abgab, welches nach einiger Zeit krystallinisch erstarrte. Dasselbe bestand ebenfalls nur aus Ephedrin-Phenylpropylenoxyd, welches der Methylierung entgangen war.

Aus diesem Verhalten geht -hervor, daß das Einwirkungsprodukt des Jodmethyls auf das Ephedrin-Phenylpropylenoxyd den Charakter eines quaternären Jodids trägt, und daß das Ephedrin-Phenylpropylenoxyd selbst daher wohl als eine tertiäre Base anzusprechen ist.

Um direkt eine vollständige Methylierung des EphedrinPhenylpropylenoxyds zu erzielen, wurde eine weitere Menge dieser 
Base in methylalkoholischer Lösung mit überschüssigem Jodmethyl 5 Stunden lang im Einschmelzrohr auf $100^{\circ}$ erhitzt. Die beim freiwilligen Verdunsten dieses Reaktionsproduktes restierende krystallinische Masse löste sich nur sehr schwer in heißem Wasser. Beim Erkalten der Lösung schieden sich schwach gelblich gefärbte, bei $164^{\circ}$ schmelzende Nadeln aus. Das aus diesem Jodid durch Umsetzen mit Chlorsilber gewonnene Chlorid bildete farblose, in Wasser ziemlich schwer lösliche, bei $165-167^{\circ}$ schmelzende Nadeln.

0,2965 $\mathrm{g}$ lieferten $0,1197 \mathrm{~g} \mathrm{AgCl}$.

$$
\begin{array}{cc}
\text { Gefunden: } & \text { Berechnet für } \underset{\mathrm{C}}{\mathrm{C}} \mathbf{\mathrm { C }} \mathrm{H}_{25} \mathrm{NO}_{2} \cdot \mathrm{CH}_{3} \mathrm{Cl} \text { : } \\
\mathrm{Cl} & \mathbf{1 0 , 1 4}
\end{array}
$$

Das aus dem Filtrat dieser Chlorbestimmung zurückgewonnene Chlorid bildete ebenso wie das Ausgangsmaterial farblose, bei $165^{\circ}$ schmelzende Nadeln.

Nach den vorstehenden Beobachtungen sind die Eigenschaften des bei der Methylierung des Ephedrin-Phenylpropylenoxyds gebildeten Additionsproduktes, je nach den angewendeten Versuchsbedingungen, verschieden. Zur Bestätigung dieser auffallenden Beobachtung wurde die Methylierung nach beiden Methoden wiederholt. Das Resultat war jedoch das gleiche.

Das bei achttägiger direkter Einwirkung von Jodmethyl bei gewöhnlicher Temperatur gebildete Jodid resultierte bein Umkrystallisieren in würfelähnlichen, bei $183^{\circ}$ schmelzenden Krystallen.

$$
\begin{aligned}
& \text { 0,139 g lieferten 0,0731 g AgJ. } \\
& \text { Gefunden: } \quad \text { Berechnet für } \mathrm{C}_{19} \mathrm{H}_{25} \mathrm{NO}_{2} \cdot \mathrm{CH}_{3} \mathrm{~J} \text { : } \\
& \text { J 28,43 28,77 }
\end{aligned}
$$

Das aus diesem Jodid durch Umsetzen mit Chlorsilber erhaltene Chlorid krystallisierte in durchsichtigen, flachen, bei $203^{\circ}$ schmelzenden Nadeln.

Das in methylalkoholischer Lösung im Einschmelzrohre gewonnene Jodid krystallisierte in Nadeln, die bei $164-165^{\circ}$ schmolzen.

$$
\begin{aligned}
& 0,1665 \mathrm{~g} \text { lieferten } 0,0855 \mathrm{~g} \text { AgJ. } \\
& \text { Gefunden: } \quad \text { Berechnet für } \mathrm{C}_{19} \mathrm{H}_{25} \mathrm{NO}_{2}, \mathrm{CH}_{3} \mathrm{~J} \text { : } \\
& \text { J } 28,73 \quad 28,77
\end{aligned}
$$

Das aus diesem Jodid erhaltene Chlorid bildete farblose, bei $165^{\circ}$ schmelzende Nadeln.

Das entsprechende Platindoppelsalz bildete orangefarbene, bei $188^{\circ}$ schmelzende Nadeln. 
A. Eberhard: Ephedrin und verwandte Verbindungen.

\section{$0,0737 \mathrm{~g}$ enthielten $0,0144 \mathrm{~g}$ Pt.}

$\begin{array}{cc}\text { Gefunden: } & \text { Berechnet fuir } \underset{19}{\left(\mathrm{C}_{19} \mathrm{H}_{25} \mathrm{NO}_{2} \cdot\left(\mathrm{CH}_{3} \mathrm{Cl}\right)_{2} \mathrm{PtCl}_{4} \text { : }\right.} \text { Pt } 18,86\end{array}$

Wie aus vorstehenden Daten und aus nachstehender $\mathrm{Zu}$ sammenstellung hervorgeht, entstehen bei der Methylierung des Ephedrin-Phenylpropylenoxyds, je nach den angewendeten Versuchsbedingungen, zwei isomere Produkte. Wodurch diese Isomerie bedingt wird, etwa durch eine molekulare Umlagerung des Ephedrin- oder Phenylpropylenoxydrestes, mag zunächst dahingestellt bleiben.

\begin{tabular}{|c|c|c|}
\hline Methylierung: & $\begin{array}{l}\text { direkt bei gewöhn- } \\
\text { licher Temperatur }\end{array}$ & $\begin{array}{c}\text { in Methylalkohol } \\
\text { bei } 100^{\circ}\end{array}$ \\
\hline $\begin{array}{l}\mathrm{C}_{19} \mathrm{H}_{25} \mathrm{NO}_{2} \cdot \mathrm{CH}_{3} \mathrm{~J} \ldots \ldots \ldots \\
\mathrm{C}_{19} \mathrm{H}_{25} \mathrm{NO}_{2} \cdot \mathrm{CH}_{3} \mathrm{Cl} \ldots \ldots \ldots \\
\left(\mathrm{C}_{19} \mathrm{H}_{23} \mathrm{NO}_{2} \cdot \mathrm{CH}_{3} \mathrm{Cl}\right)_{2} \mathrm{PtCl}{ }_{4}\end{array}$ & $\begin{array}{c}\text { Schmelzp. } 183^{\circ} \\
\text { Schmelzp. } 203^{\circ} \\
-\end{array}$ & $\begin{array}{l}\text { Schmolzp. } 164^{\circ} \\
\text { Schmelzp. } 165-167^{\circ} \\
\text { Schmelzp. } 185^{\circ}\end{array}$ \\
\hline
\end{tabular}

Benzoylderivat. Zur Ermittelung der Anzahl der im Molekül der fraglichen Base enthaltenen Hydroxylgruppen wurde dieselbe der Benzoylierung nach dem Verfahren von S c hot te n-B a u m a n unterworfen. Beim wiederholten Ausschütteln dës alkalischen Reaktionsproduktes mit Aether und Verdunstenlassen der vereinigten Auszüge verblieb eine ölige Masse, welche nicht zur Krystallisation gebracht werden konnte. Es wurde daher die Benzoylierung in derselben Weise wiederholt, dabei wurden jedoch die ersten Aetherausschüttelungen gesondert verdunstet. Hierbei restierte zunächst zwar auch nur ein öliges Liquidum, welches jedoch nach mehrtägiger Aufbewahrung krystallinisch erstarrte. Letzteres war bei dem Verdunstungsrückstande der letzten Aetherausschüttelungen nicht der Fall.

Nach dem Abpressen und Umkrystallisieren aus verdünntem Alkohol lieferte jene krystallinische Masse weiße, bei $141-142^{\circ}$ schmelzende, nadelförmige Krystalle.

$0,139 \mathrm{~g}$ lieferten $0,3969 \mathrm{~g} \mathrm{CO}_{2}$ und $0,0819 \mathrm{~g} \mathrm{H}_{2} \mathrm{O}$.

\begin{tabular}{cccc}
\multicolumn{2}{c}{ Gefunden: } & \multicolumn{3}{c}{ Berechnet für } \\
C & $\mathbf{7 7 , 8 8}$ & $\mathrm{C}_{19} \mathrm{H}_{24} \mathrm{NO}\left(\mathrm{O} . \mathrm{C}_{7} \mathrm{H}_{5} \mathrm{O}\right):$ & $\mathrm{C}_{19} \mathrm{H}_{23} \mathrm{~N}\left(\mathrm{O} . \mathrm{C}_{7} \mathrm{H}_{8} \mathrm{O}\right)_{2}$ : \\
$\mathrm{H}$ & $\mathbf{6 , 6 9}$ & $\mathbf{7 7 , 3 7}$ & $\mathbf{7 8 , 0 7}$ \\
& $\mathbf{7 , 1 9 4 7}$ & $\mathbf{7 , 2 5}$ & 6,56
\end{tabular}

$0,1947 \mathrm{~g}$ verbrauchten zur Verseifung $1,53 \mathrm{ccm} 1 / 2-\mathrm{N}$.-Kalilauge $=0,04392 \mathrm{~g} \mathrm{KOH}$.

Berechnet für

$$
\begin{array}{cc}
\mathrm{C}_{19} \mathrm{H}_{24} \mathrm{NO}\left(\mathrm{O} . \mathrm{C}_{7} \mathrm{H}_{5} \mathrm{O}\right): & \mathrm{C}_{19} \mathrm{H}_{23} \mathrm{~N}\left(\mathrm{O} . \mathrm{C}_{7} \mathrm{H}_{5} \mathrm{O}\right)_{2}: \\
\mathrm{KOH} & 0,02709
\end{array}
$$


Aus diesen analytischen Daten geht hervor, daß in der analysierten Verbindung ein Dibenzoylderivat der Verbindung $\mathrm{C}_{10} \mathrm{H}_{25} \mathrm{NO}_{2}$ vorlag, daß somit, unter Berücksichtigung des Charakters als tertiäre Base, die beiden Sauerstoffatome in derselben als Hydroxylgruppen enthalten sein mußten.

\section{Versuche zur Darstellung von Inaktivem Ephedrin, bzw. Pseudoephedrin.}

Als Ausgangsmaterial für diese Versuche diente das AethylPhenylketon, eine Verbindung, welche bereits früher von A. Goeh $\mathrm{ring}^{1}$ ) und von F. W. Calli e $B^{2}$ ) auf Veranlassung von E. S ch midt zu diesem Zwecke, wenn auch nicht mit dem gewünschten Erfolge, verwendet worden war. Es wurden diese Versuche wiederholt, da immerhin die Möglichkeit vorlag, daß durch Aenderung der Versuchsbedingungen doch die Synthese des Ephedrins, bzw. einer diesem Alkaloid nahestehenden, optisch inaktiven Base realisiert werden könnte.

Das Aethyl-Phenylketon: $\mathrm{C}_{6} \mathrm{H}_{5}-\mathrm{CO}-\mathrm{CH}_{2}-\mathrm{CH}_{3}$, wurde $\mathrm{zu}$ diesem Zwecke zunüchst einesteils in $\alpha$-Amidoäthyl-Phenylketon: $\mathrm{C}_{6} \mathrm{H}_{5}-\mathrm{CO}-\mathrm{CH}\left(\mathrm{NH}_{2}\right)-\mathrm{CH}_{3}$, anderenteils in $\alpha$-Bromäthyl-Phenylketon: $\mathrm{C}_{6} \mathrm{H}_{5}-\mathrm{CO}-\mathrm{CHBr}-\mathrm{CH}_{3}$, verwandelt. Das $\alpha$-AmidoäthylPhenylketon sollte dann methyliert und weiter durch Reduktion in ein Carbinol verwandelt werden. Das $\alpha$-Bromäthyl-Phenylketon wurde dagegen zunächst mit Methylamin in Reaktion versetzt und das hierbei gebildete Produkt hierauf der Reduktion unterworfen.

\section{Versuche mit $\alpha$-Amidoäthyl-Phenglketon.}

Zur Darstellung des $\alpha$-Amidoäthyl-Phenylketons: $\mathrm{C}_{8} \mathrm{H}_{5}-\mathrm{CO}$ $\mathrm{CH}\left(\mathrm{NH}_{2}\right)-\mathrm{CH}_{3}$, diente das nach den Angaben von $\mathrm{Cl}$ a i se $\mathrm{n}$

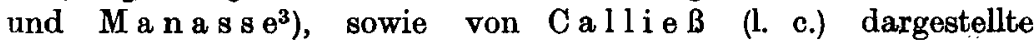
$\alpha$-Nitrosoäthyl-Phenylketon: $\mathrm{C}_{6} \mathrm{H}_{5}-\mathrm{CO}-\mathrm{CH}(\mathrm{NO})-\mathrm{CH}_{3}$. Die Reduktion letzterer Verbindung erfolgte nach $\mathrm{B}$ e h r - B r e g o w s k i) und nach $\mathrm{C}$ a lli e $\mathrm{B}$ durch Schütteln mit einer Lösung von Zinnchlorür in rauchender Salzsäure. Das Hydrochlorid des Amidoketons bildete farblose oder blätterige, entsprechend den Angaben von $\mathrm{C}$ a 11 i e $B$, bei $179^{\circ}$ schmelzende Krystalle.

$0,3649 \mathrm{~g}$ lieferten $0,283 \mathrm{~g}$ AgCl.

Gefunden: Berechnet für $\mathrm{C}_{6} \mathrm{H}_{5}-\mathrm{CO}-\mathrm{CH}\left(\mathrm{NH}_{2}\right)-\mathrm{CH}_{3}, \mathrm{HCl}$ :

$\begin{array}{lll}\text { Cl } 19,2 & 19,1 \quad \text { (Schluß folgt.) }\end{array}$

1) Dieses Archiv 1909, 142.

2) Ibidem 1912, 141.

3) Ber. d. d. chem. Ges. 1889, 256.

4) Ibidem 1897, 1521 . 
Das aus diesem Hydrochlorid durch Sodalösung abgeschiedene und durch Aether ausgeschüttelte Amidoketon schmolz bei $112-114^{0}$.

Das Amidopropiophenon wurde zunächst, unter Anlehnung an das von $G a d a m e r$ und $K u n t z e^{1}$ ) für die Methylierung des Bulbocapnins benutzte Verfahren, mit $D$ i a z o methan behandelt, ohne jedoch dabei zu einem Methylierungsprodukt zu gelangen.

Einwirkung von Dimethylsulfat.

Das Verhalten des Dimethylsulfats gegen AmidoäthylPhenylketon ist bereits von Callie B (l. c.), jedoch ohne den gewünschten Erfolg, studiert worden. Es wurden daher diese Versuche wiederholt. Das Hydrochlorid des Amidoketons wurde zu diesem Zweck zunächst mit der vierfachen Menge Dimethylsulfat zusammengebracht und das Gemisch 8 Tage lang, unter häufigem Umschütteln, in einem verschlossenen Gefäße bei gewöhnlicher Temperatur aufbewahrt. Das aus diesem Produkt dargestellte, in kompakten Nadeln krystallisierende Platindoppelsalz erwies sich jedoch als das Platinat des unveränderten Amidopropiophenons.

$0,330 \mathrm{~g}$ enthielten $0,0918 \mathrm{~g} \mathrm{Pt}$.

Gefunden: Berechnet für $\left[\mathrm{C}_{6} \mathrm{H}_{5}-\mathrm{CO}-\mathrm{CH}\left(\mathrm{NH}_{2}\right)-\mathrm{CH}_{3}, \mathrm{HCl}\right]_{2} \mathrm{PtCl}$ :
$\mathrm{Pt} \quad 27,56$

Es wurde daher das obige Gemisch 6 Stunden lang mit aufgesetztem Steigrohr auf dem Wasserbade erhitzt. Das aus einer Probe dieses Reaktionsproduktes dargestellte Platindoppelsalz bildete nadelförmige, bei $215^{\circ}$ schmelzende Krystalle. Dieselben bestanden aus dem Platinat des quaternären Chlorids, welches nach $\mathrm{C}$ a 11 i e $\beta$ bei $21^{\circ}$ schmilzt.

$0,2068 \mathrm{~g}$ enthielten $0,0512 \mathrm{~g} \mathrm{Pt}$.

$$
\text { Gefunden: Berechnet für }\left[\mathrm{C}_{6} \mathrm{H}_{5}-\mathrm{CO}-\mathrm{C}_{2} \mathrm{H}_{4} \cdot \mathrm{N}\left(\mathrm{CH}_{3}\right)_{3} \mathrm{Cl}_{2}\right]_{2} \mathrm{PtCl}_{4} \text { : }
$$

Zum Nachweis weiterer Methylierungsprodukte sekundärer, bzw. tertiärer Natur, wurde der Rest des Reaktionsprodukts zunächst durch Eindampfen möglichst von Dimethylsulfat befreit, der Rückstand alsdann in Wasser gelöst und die mit Natronlauge alkalisch gemachte Lösung (L) hierauf mit Aether und schließlich mit Chloroform ausgeschüttelt. Beim Verdunsten dieser Lösungsmittel verblieb nur ein geringer, braun gefärbter Rückstand, der

1) Dieses Archiv 1911, 615.

Arch. d. Pharm. CCLIII. Bde. 2. Heft. 
sich nur zum Teil in heißer verdünnter Salzsäure löste. Aus dieser Lösung schieden sich einige kurze, bei $123^{\circ}$ schmelzende Nadeln aus. $\mathrm{Zu}$ deren Identifizierung wurde die ganze Menge dieser salzsauren Lösung in ein Golddoppelsalz verwandelt. Letzteres krystallisierte in glänzenden, bei $150^{\circ}$ schmelzenden Blättchen. Nach dem Schmelzpunkt und dem Goldgehalt lag in diesem Aurat ein Doppelsalz des D i m e th y la m i d o-P r o p i ophenons ror.

$0,1495 \mathrm{~g}$ enthielten $0,0569 \mathrm{~g}$ Au.

Gefunden: Berechnet für $\mathrm{C}_{6} \mathrm{H}_{5}-\mathrm{CO}-\mathrm{C}_{2} \mathrm{H}_{4} \cdot \mathrm{N}\left(\mathrm{CH}_{3}\right)_{2}, \mathrm{HCl}+\mathrm{AuCl}_{3}$ : $\mathrm{Au} \quad 38,06$ 38,10

Aus den Mutterlaugen dieses Golddoppelsalzes konnte kein weiteres Aurat, welches auf das Vorhandensein eines Monomethylamido-Propiophenons hingewiesen hätte, isoliert werden.

Die mit Aether, bzw. Chloroform ausgeschüttelte alkalische Lösung (L) enthielt Trimeth y lamido-Propiophen on, welches als Hauptprodukt bei obiger Methylierung gebildet war. Das betreffende Aurat bildete glänzende, schwer lösliche Blättchen, welche entsprechend den Angaben von Callie $B$ bei 153 ${ }^{\circ}$ schmolzen.

$0,131 \mathrm{~g}$ enthielten $0,0483 \mathrm{~g} \mathrm{Au}$.

Gefunden: Berechnet für $\mathrm{C}_{6} \mathrm{H}_{5}-\mathrm{CO}-\mathrm{C}_{2} \mathrm{H}_{4} \cdot \mathrm{N}\left(\mathrm{CH}_{3}\right)_{3} \mathrm{Cl}, \mathrm{AuCl}_{3}$ : Au 37,10 37,11

Bei der Einwirkung von Dimethylsulfat auf Amidopropiophenon war somit, neben wenig Dimeth yla mid o-Propiophenon, nur quaternäres Trimethylamido-Propiophe n o n gebildet worden.

Einwirkung von Jodmethyl.

Die Einwirkung von Jodmethyl auf Amidopropiophenon ist ebenfalls bereits von Ca 11 i e B (l. c.) zum Gegenstand der Untersuchung gemacht. Hierbei wurde ermittelt, daß das Amidopropiophenon bei gewöhnlicher Temperatur in methylalkoholischer Lösung durch Jodmethyl nur in ein Dimethyl- und besonders in ein Trimethylderivat ühergeführt wird. Das gleiche ist auch der Fall bei der Methylierung nach $N$ ölt in $g^{1}$ ), in Gegenwart von Soda, wie die Eigenschaften und die Zusammensetzung des aus dem Reaktionsprodukt dargestellten Chlorids, Aurats und Platinats lehrten.

1) Ber. d. d. chem. Ges. 1891, 563. 
Chlorid: farblose, bei $196-198^{\circ}$ schmelzende Nadeln; Chlorgehalt 15,85\%. A u r a t: gelbe, bei $156^{\circ}$ schmelzende Blättchen; Goldgehalt 37,76\%. P l a t in a t: schwer lösliche, rotgelbe, bei $217-218^{\circ}$ schmelzende Nadeln; Platingehalt 25,35\%.

\section{Versuche mit $\alpha$-Amidoäthyl-Phenylcarbinol.}

Da auch die erneuten Methylierungsversuche des $\alpha$-Amidoäthyl-Phenylketons nicht zu dem gewünschten Resultat geführt hatten und die hierbei nur erhaltenen tertiären und quaternären Methylierungsprodukte nicht ohne Spaltung durch Natriumamalgam zu Carbinolen reduzierbar sind, wurde die Einwirkung des Jodmethyls auf das $\alpha$-Amidoäthyl-Phenylcarbinol, welche ebenfalls bereits von Calli e B (l. c.) zur Ausführung gelangte, wiederholt.

Das hierzu erforderliche $\alpha$-Amidoäthyl-Phenylcarbinol : $\mathrm{C}_{6} \mathrm{H}_{5}-\mathrm{CH} . \mathrm{OH}-\mathrm{CH} . \mathrm{NH}_{2}-\mathrm{CH}_{3}$, wurde nach den Angaben von $\mathrm{K} \circ \mathrm{h} \mathrm{n}^{1}$ ), E $\mathrm{mde} \mathrm{e}^{2}$ ) und CallieB (l. c.) durch Reduktion des $\alpha$-Amidoäthyl-Phenylketons dargestellt. Das gewonnene Hydrochlorid bildete nadelförmige, im Einklang mit den Angaben von Ca 11 i e B, bei $191^{\circ}$ schmelzende Krystalle.

Zur Methylierung wurde das freie Carbinol mit überschüssigem Jodmethyl geschüttelt. Nach erfolgter Lösung trat eine lebhafte Reaktion ein, nach deren Beendigung das Reaktionsprodukt zum größten Teil zu einer gelblichen, krystallinischen Masse erstarrte. Zur-Trennung der in derselben enthaltenen Verbindungen wurde das überschüssige Jodmethyl verdunstet, der Rückstand in Wasser gelöst, die Lösung mit feuchtem Silberoxyd in geringem Ueberschuß versetzt und das Gemisch wiederholt mit Aether ausgeschüttelt.

Beim Verdunsten dieser Aetherauszüge verblieb nur eine verhältnismäßig geringe Menge eines blaßgelben öligen Liquidums, welches anscheinend nur aus tertiärer Base bestand. Dasselbe löste sich in Jodmethyl leicht auf zu einer Flüssigkeit, welche unter Selbsterwärmung alsbald krystallinisch erstarrte. Dieses Produkt lieferte nach dem Abpressen und Umkrystallisieren aus Alkohol schwer lösliche, zu Warzen gruppierte, farblose Nadeln, die bei $221^{\circ}$ schmolzen.

$0,3447 \mathrm{~g}$ lieferten $0,252 \mathrm{~g} \mathrm{AgJ}$.

Gefunden: Berechnet für $\mathrm{C}_{6} \mathrm{H}_{5}-\mathrm{CH} . \mathrm{OH}-\mathrm{C}_{2} \mathrm{H}_{4} \cdot \mathrm{N}\left(\mathrm{CH}_{3}\right)_{3} \mathrm{~J}$ :

J 39,51 39,56

1) Monatsh. 1907, 336.

2) Dieses Archiv 1909, 136. 
Die mit Aether ausgeschüttelte, die quaternäre Base enthaltende Lösung wurde mit Salzsäure angesäuert und zur Darstellung eines Platin- und Golddoppelsalzes verwendet.

Das Platindoppelsalz bildete rotgelbe, in Wasser schwer lösliche Nadeln, welche im Einklang mit den Angaben von Ca 11 i e $B$ bei $247^{\circ}$ schmolzen.

Das Golddoppelsalz krystallisierte in gelben, in Wasser schwer Iöslichen Nadeln, die bei $172-173^{\circ}$ schmolzen. Callie B fand den Schmelzpunkt dieses Aurats bei 171-1720.

$0,4347 \mathrm{~g}$ enthielten 0,1602 $\mathrm{g}$ Au.

Gefunden: Berechnet für $\mathrm{C}_{6} \mathrm{H}_{5}-\mathrm{CH} . \mathrm{OH}-\mathrm{C}_{2} \mathrm{H}_{4} \cdot \mathrm{N}\left(\mathrm{CH}_{3}\right)_{3} \mathrm{Cl}, \mathrm{AuCl}_{3}$ : Au 36,85 36,97

Das aus diesem Golddoppelsalz dargestellte Chlorid bildete zunächst nur eine dickflüssige Masse, die erst bei Winterkälte allmählich in ein krystallinisches Produkt überging. Durch Behandeln desselben mit wenig Aceton ließen sich dann hieraus kurze, nadelförmige, bei $204^{\circ}$ schmelzende Krystalle isolieren.

Die bei der Methylierung des $\alpha$-Amidoäthyl-Phenylcarbinols erhaltenen, optisch inaktiven quaternären Verbindungen sind isomer mit den entsprechenden, optisch aktiven Derivaten des Ephedrins und Pseudoephedrins, wie aus nachstehender Zusammenstellung hervorgeht.

\begin{tabular}{|c|c|c|c|}
\hline & aus Ephedrin & $\begin{array}{l}\text { aus Pseudo- } \\
\text { ephedrin }\end{array}$ & $\begin{array}{l}\text { aus Amidoäthyl- } \\
\text { Phenylcarbinol }\end{array}$ \\
\hline Jodid & Schmp. $204^{\circ}$ & Schmp. $205^{\circ}$ & Schmp. $220^{\circ}$ \\
\hline Chlorid .... & Schmp. $230^{\circ}$ & - & Schmp. $204^{\circ}$ \\
\hline Aurat ..... & Schmp. 188-1910 & Schmp. $194-195^{\circ}$ & $\begin{array}{c}\text { Schmp. 172-173 } \\
\text { (nach Callie } \\
\left.171-172^{\circ}\right)\end{array}$ \\
\hline Platinat & Schmp. 247-250 & Schmp. 204-2050 & Schmp. $247^{\circ}$ \\
\hline
\end{tabular}

Zum Vergleich mit vorstehenden Verbindungen wurde auch das Methylierungsprodukt des Amido-Benzylmethylc a r b i n o ls: $\mathrm{C}_{6} \mathrm{H}_{5}-\mathrm{CH} . \mathrm{NH}_{2}-\mathrm{CH} . \mathrm{OH}-\mathrm{CH}_{3}$, dargestellt. Auch dieses Amidoketon lieferte bei der Einwirkung von Jodmethyl, neben tertiärer Base, im wesentlichen nur quaternäres Jodid, wie bereits früher von $\mathrm{E}$. $\mathrm{S} \mathrm{c} \mathrm{h} \mathrm{m}$ i d $\mathrm{t}^{1}$ ) konstatiert worden war.

Das Hydrojodid des Dimethylamido-Benzylcarbinols bildet farblose, prismatische, bei $220-221^{\circ}$ schmelzende Krystalle.

1) Apoth.-Ztg. 191 1, No. 37. 
$0,573 \mathrm{~g}$ lieferten $0,4379 \mathrm{~g}$ AgJ.

Gefunden: Berechnet für $\mathrm{C}_{6} \mathrm{H}_{5}-\mathrm{CH} . \mathrm{N}\left(\mathrm{CH}_{3}\right)_{2}-\mathrm{CH} . \mathrm{OH}-\mathrm{CH}_{3}$, HJ :
$\mathbf{J} 41,31$

Das Chlorid des Trimethylamido-Benzylc a r b in ols: $\mathrm{C}_{6} \mathrm{H}_{5}-\mathrm{CH} . \mathrm{N}\left(\mathrm{CH}_{3}\right)_{3} \mathrm{Cl}-\mathrm{CH} . \mathrm{OH}-\mathrm{CH}_{3}+\mathrm{H}_{2} \mathrm{O}$, schied sich aus verdünntem Alkohol in kleinen, würfelförmigen Krystallen aus, welche wasserfrei bei $196^{\circ}$ schmolzen. H. E m d $\theta^{1}$ ) fand den Schmelzpunkt bei $196-197^{\circ}$.

$0,289 \mathrm{~g}$ verloren bei $100^{\circ} 0,0208 \mathrm{~g}$ an Gewicht und erforderten zur Fällung $11,56 \mathrm{ccm} 1 / 10-\mathrm{N}$.-Silbernitratlösung.

Berechnet für

\begin{tabular}{|c|c|}
\hline Gefunden: & $\mathrm{C}_{8} \mathrm{H}_{5}-\mathrm{CH} . \mathrm{N}\left(\mathrm{CH}_{3}\right)_{3} \mathrm{Cl}-\mathrm{CH} . \mathrm{OH}-\mathrm{CH}_{3}+\mathrm{H}_{2} \mathrm{O}:$ \\
\hline $\mathrm{H}_{2} \mathrm{O} \quad 7,20$ & 7,27 \\
\hline Cl $\quad 14,18$ & 14,32 \\
\hline
\end{tabular}

Für das A u rat fand E. S ch midt (l. c.) den Schmelzpunkt bei $150-151^{\circ}$, für das P lat in a t bei $232-234^{\circ}$.

\section{Versuche mit $\alpha$-Bromäthyl-Phenylketon.}

Das $\alpha$-Bromäthyl - Phenylketon: $\mathrm{C}_{6} \mathrm{H}_{5}-\mathrm{CO}-\mathrm{CHBr}-\mathrm{CH}_{3}$, welches für diese Methylierungsversuche Verwendung fand, wurde nach den Angaben von Chr. S $\mathrm{ch} \mathrm{mi} \mathrm{t}^{2}$ ) durch Einwirkung von Brom auf Aothyl-Phenylketon dargestellt.

Die Einwirkung dieses Bromids auf Methylamin ist in absolut alkoholischer Lösung bereits von A. G o e h ring ${ }^{3}$ ) zum Gegenstand einer Untersuchung gemacht. Es wurde hierbei zwar die Bildung einer Verbindung der Formel $\mathrm{C}_{6} \mathrm{H}_{5}-\mathrm{CO}-\mathrm{CH}\left(\mathrm{NHCH}_{3}\right)-\mathrm{CH}_{3}$ konstatiert, jedoch war die Ausbeute daran eine sehr geringe. Es mußte daher die nächste Aufgabe sein, die Versuchsbedingungen zu ermitteln, unter denen dieses Reaktionsprodukt in größerer Menge gebildet wird.

a) Einwirkung von Methylamin in alkoholischer L ö s u ng.

Zur Orientierung über den Reaktionsverlauf wurden zunächst $5 \mathrm{~g}$ Brompropiophenon in einer mit Glasstopfen verschlossenen Flasche in der gleichen Gewichtsmenge absolutem Alkohol gelöst, diese Lösung dann mit $33 \%$ absolut alkoholischer Methylaminlösung im Ueberschuß versetzt und das Gemisch hierauf verschlossen 14 Tage lang bei gewöhnlicher Temperatur aufbewahrt.

1) Ber. d. d. chem. Ges. 1910, 1728.

2) Ber. d. d. chem. Ges. 1889, 3250.

3) Dieses Archiv 1909, 146. 
Das anfangs blaßgelb gefärbte Gemisch nahm hierbei sehr bald eine intensiv gelbrote Färbung an. Eine freiwillige Erwärmung trat hierbei nur in geringem Umfange ein. Das Reaktionsprodukt wurde alsdann der freiwilligen Verdunstung überlassen, der hierbei verbleibende, braun gefärbte Rückstand hierauf mit heißem, salzsäurehaltigem Wasser ausgezogen und die erzielte Lösung im Exsikkator verdunstet. Hierbei verblieb eine teerartige, in heißem Wasser nur teilweise lösliche Masse.

Der wässerige Auszug dieses Produkts wurde dann mit Chlorsilber umgesetzt und die immer noch stark rot gefärbte Lösung zur Entfärbung mit Quecksilberchloridlösung versetzt. Nach dem Abfiltrieren der hierdurch ausgeschiedenen braunen Flocken und Entfernen des Quecksilbers durch Schwefelwasserstoff resultierte eine nur blaßgelb gefärbte Flüssigkeit, die nach dem Eindampfen auf ein kleines Volum zur Darstellung eines Golddoppelsalzes diente. Letzteres war in Wasser und in verdünntem Alkohol bei gewöhnlicher Temperatur schwer löslich. Durch wiederholtes Umkrystallisieren zunächst aus heißem verdünnten Alkohol, dann aus viel heißem Wasser gelang es, dasselbe in kleine blätterige oder nadelförmige Krystalle, die bei $168,5-169^{\circ}$ schmolzen, überzuführen.

1. $0,1310 \mathrm{~g}$ enthielten $0,0486 \mathrm{~g}$ Au.

2. $0,2433 \mathrm{~g},, \quad 0,0902 \mathrm{~g}$ Au.

3. $0,1940 \mathrm{~g}$ einer weiteren Krystallisation enthielten $0,0708 \mathrm{~g} \mathrm{Au}$.

$$
\begin{aligned}
& \text { Gefunden: } \\
& \text { 1. } 2 . \quad 3 \text {. } \\
& \text { Au } \quad 37,10 \quad 37,07 \quad 36,50 \\
& \mathrm{C}_{6} \mathrm{H}_{5}-\mathrm{CO}-\mathrm{C}_{2} \mathrm{H}_{4} \cdot \mathrm{NH} . \mathrm{CH}_{3}, \mathrm{HCl}+\mathrm{AuCl}_{3} \text { : } \\
& \text { Au 39,18 } \\
& \mathrm{C}_{6} \mathrm{H}_{5}-\mathrm{CO}-\mathrm{C}_{2} \mathrm{H}_{4} \cdot \mathrm{N}\left(\mathrm{CH}_{3}\right)_{3} \mathrm{Cl}, \mathrm{AuCl}_{3} \text { : } \\
& \text { Au 37,11 }
\end{aligned}
$$

Das diesen Auraten entsprechende Platinat bildete schwer lösliche, gelbrote, bei $186-186,5^{\circ}$ schmelzende Blättchen (I). Aus der Mutterlauge dieses Platinats (I) resultierten noch kleine, rotgelbe, säulenförmige Krystalle (II), welche etwas leichter in Wasser löslich waren. Dieselben schmolzen bei $188^{\circ}$.

1. 0,2524 $\mathrm{g}$ enthielten $0,0610 \mathrm{~g} \mathrm{Pt}$.

2. $0,2147 \mathrm{~g} \quad, \quad 0,0528 \mathrm{~g}$ Pt. Gefunden: $\quad$ Berechnet für
1. 2.

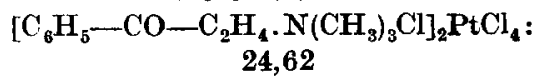

Pt $\quad 24,17 \quad 24,60$ 
Nach den Ergebnissen der vorstehenden Analysen war unter obigen Versuchsbedingungen die Bildung einer Base der Formel: $\mathrm{C}_{6} \mathrm{H}_{5}-\mathrm{CO}-\mathrm{C}_{2} \mathrm{H}_{4}\left(\mathrm{NH} . \mathrm{CH}_{3}\right)$, wohl kaum erfolgt. Die gefundenen Werte stimmen vielmehr mit denen einer quaternären Verbindung: $\mathrm{C}_{6} \mathrm{H}_{5}-\mathrm{CO}-\mathrm{C}_{2} \mathrm{H}_{4} \cdot \mathrm{N}\left(\mathrm{CH}_{3}\right)_{9} \mathrm{Cl}$, überein, jedoch weisen die bezüglichen Schmelzpunkte erhebliche Differenzen auf; Aurat $169: 152,5$, Platinat 188: 215.

b) Einwirkung von Methylamin in Benzollösung.

Die Einwirkung des Brompropiophenons auf Methylamin ist bei $0^{0}$ in Benzollösung bereits von E. $S$ ch m i d t $t^{1}$ ) zur Ausführung gebracht. Die Ausbeute an Methylamido-Propiophenon, welche damals unter diesen Bedingungen erzielt wurde, war jedoch nur eine geringe, so daß bei den weiteren Versuchen absolutalkoholische Methylaminlösung bei $0^{0}$ benutzt wurde (Privatmitteilung).

Bei der Wiederholung dieser Versuche wurde zunächst ebenfalls Benzollösung verwendet und dabei nach den Angaben von Emde und $R \mathrm{unn}^{2}$ ) über die Methylierung des Bromphenylacetons verfahren.

$5 \mathrm{~g}$ Brompropiophenon wurden zu diesem Zwecke mit $12 \mathrm{~g}$ Benzol gemischt und diese Flüssigkeit in einer Kältemischung abgekühlt, bis ein Teil des Benzols zu erstarren anfing. Hierauf wurde aus einem Scheidetrichter allmählich eine stark abgekühlte Lösung von Methylamin in Benzol von 5,77 \% zutropfen gelassen, bis das auskrystallisierte Benzol wieder geschmolzen war. Zu der von neuem bis zur Ausscheidung von festem Benzol gekthhlten Lösung wurde dann Methylaminlösung abermals zugefügt, bis sich das Benzol wieder verflüssigte. Diese Operationen wurden so lange fortgesetzt, bis 29,3 $\mathrm{g}$ der 5,77\%igen Methylaminlösung, entsprechend $2 \mathrm{Mol}$. $\mathrm{NH}_{2} . \mathrm{CH}_{3}$ auf $1 \mathrm{Mol} . \mathrm{C}_{6} \mathrm{H}_{5}-\mathrm{CO}-\mathrm{C}_{2} \mathrm{H}_{4} \mathrm{Br}$, eingetragen waren. Nach erneuter kurzer Abkühlung wurde hierauf das ausgeschiedene Methylaminhydrobromid von der gelb gefärbten Flüssigkeit durch Absaugen getrennt, mit wenig kaltem Benzol ausgewaschen und die Benzollösung dann viermal mit stark abgekühlter Salzsäure von $12,5 \%$ ausgeschüttelt. Diese Lösungen wurden hierauf verdampft und der sirupartige Rückstaind in den Exsikkator gestellt, worin ex zu einer krystallinischen Masse (M) erstarrte.

1) Apoth.-Ztg. 1911, 368.

2) Dieses Archiv 1911, 368. 
Nachdem durch Behandeln mit wenig kaltem Aceton die färbenden Beimengungen entfernt waren, wurde die Masse (M) in Wasser gelöst und diese Lösung dann mit frisch gefälltem Chlorsilber, zur Entfernung des Broms, behandelt. Goldchlorid rief in dieser Lösung eine ölige Ausscheidung hervor, welche nicht zur Krystallisation gebracht werden konnte. Das Gold wurde daher aus der durch Zusatz von Alkohol erzielten Lösung durch $\mathrm{H}_{2} \mathrm{~S}$ wieder entfernt, dieselbe hierauf eingedampft und mit Platinchloridlösung versetzt. Hierdurch erfolgte eine reichliche Ausscheidung von blätterigen, bei 218-2240 schmelzenden Krystallen, die aus Methylaminplatinchlorid bestanden (gefunden $41,3 \% \mathrm{Pt}$ ).

Erst aus den letzten Mutterlaugen schieden sich rote, warzenförmige Krystalle aus, die bei $186^{\circ}$ schmolzen. Dieselben bestanden, wie die weitere Untersuchung derselben lehrte, aus dem Platinat des Methylamino-Propiophenons. Die Menge dieser Krystalle war jedoch im Verhältnis zu dem angewendeten Brompropiophenon nur gering. Der Versuch wurde daher unter Anwendung von $20 \mathrm{~g}$ Brompropiophenon unter den gleichen Bedingungen wiederholt, die nach dem Ausschütteln mit Salzsäure und darauffolgendes Eindampfen gewonnene krystallinische Masse (M) jedoch zunächst in möglichst wenig siedendem absoluten Alkohol gelöst. Beim Erkalten dieser Lösung schieden sich allmählich beträchtliche Mengen von Methylaminhydrochlorid ab. Die hiervon getrennte Lösung wurde hierauf verdunstet, der Rückstand in Wasser gelöst, diese Lösung mit Chlorsilber digeriert und das Filtrat, nach genügender Konzentration, mit Platinchloridlösung versetzt.

Neben blätterigem Methylaminplatinchlorid schieden sich auch hier allmählich rote, warzenförmige, bei $187^{\circ}$ unter Aufschäumen schmelzende Krystalle aus, welche leicht durch Auslesen getrennt werden konnten.

$0,4464 \mathrm{~g}$ enthielten $0,1179 \mathrm{~g}$ Pt.

Gefunden: Berechnet für $\left.\underset{\text { Pt } 26,41}{\left(\mathrm{C}_{6} \mathrm{H}_{5}-\mathrm{CO}-\mathrm{C}_{2} \mathrm{H}_{4}\right.} \cdot \mathrm{NHCH}_{3}, \mathrm{HCl}\right)_{2} \mathrm{PtCl}_{4}$ :

Das aus diesem Platinat dargestellte A u r a t schied sich zunächst ölig ab, verwandelte sich jedoch nach Verlauf von mehreren Tagen in Büschel von wohlausgebildeten, bei $120^{\circ}$ schmelzenden Nadeln.

$0,3409 \mathrm{~g}$ enthielten 0,1337 $\mathrm{g} \mathrm{Au}$.

Gefunden: Berechnet für $\mathrm{C}_{6} \mathrm{H}_{5}-\mathrm{CO}-\mathrm{C}_{2} \mathrm{H}_{4} \cdot \mathrm{NHCH}_{3}, \mathrm{HCl}+\mathrm{AuCl}_{3}$ : Au $\mathbf{3 9 , 2 2}$ 
Das $\mathrm{Hydrochlorid:} \mathrm{C}_{6} \mathrm{H}_{5}-\mathrm{CO}-\mathrm{C}_{2} \mathrm{H}_{4} \cdot \mathrm{NHCH}_{3}, \mathrm{HCl}$, bildete nach dem Umkrystallisieren aus einem Gemisch von absolutem Alkohol und Aceton farblose, warzenförmige, bei $179^{\circ}$ schmelzende Krystalle.

Wenn die Ausbeute an Methylamido-Propiophenon nach dem im vorstehenden beschriebenen Verfahren eine wenig befriedigende war, so lag dies daran, daß der größte Teil des angewendeten Brompropiophenons unter diesen Versuchsbedingungen überhaupt nicht in Reaktion getreten war, sondern sich noch unverändert in dem Benzol in Lösung befand. Bei einem weiteren Versuche wurde daher das unter Eiskühlung bereitete Gemisch aus Benzol, Methylamin und Brompropiophenon vor dem Ausschütteln mit Salzsäure erst noch mehrere Stunden lang bei gewöhnlicher Temperatur stehen gelassen. Die Ausbeute an MethylamidoPropiophenon wurde hierdurch zwar wesentlich erhöht, ohne sich jedoch zu einer guten zu gestalten.

Noch otwas besser gestaltete sich die Ausbeute an dieser Verbindung, als die Einwirkung des Brompropiophenons unter sonst den gleichen Bedingungen in absolutalkoholischer Lösung und unter Benutzung von absolutalkoholischer Methylaminlösung von $33 \%$ vorgenommen wurde. Immerhin blieb jedoch auch hier ein sehr beträchtlicher Teil des angewendeten Brompropiophenons unverändert.

Das aus diesem Reaktionsprodukt gewonnene Platindoppelsalz entsprach durchaus dem aus der Benzollösung erhaltenen.

$0,3294 \mathrm{~g}$ enthielten $0,0877 \mathrm{~g}$ Pt.

Gefunden: Berechnet für $\left(\mathrm{C}_{6} \mathrm{H}_{5}-\mathrm{CO}-\mathrm{C}_{2} \mathrm{H}_{4} \cdot \mathrm{NHCH}_{3}, \mathrm{HCl}\right)_{2} \mathrm{PtCl}_{4}$ : Pt $\quad 26,62$ 26,52

Das entsprechende Golddoppelsalz schied sich zunächst ebenfalls ölig $a b$, verwandelte sich aber allmählich in büschelförmige, bei $119-120^{\circ}$ schmelzende Nadeln.

$0,4776 \mathrm{~g}$ enthielten $0,1869 \mathrm{~g} \mathrm{Au}$.

Gefunden: Berechnet für $\mathrm{C}_{6} \mathrm{H}_{5}-\mathrm{CO}-\mathrm{C}_{2} \mathrm{H}_{4} \cdot \mathrm{NHCH}_{3}, \mathrm{HCl}+\mathrm{AuCl}_{3}$ : Au 39,13 39,19

\section{Methylamino-Aethylphenylcarbinol:$$
\mathrm{C}_{6} \mathrm{H}_{5}-\mathrm{CH} . \mathrm{OH}-\mathrm{CH} . \mathrm{NH}\left(\mathrm{CH}_{8}\right)-\mathrm{CH}_{3} \text {. }
$$

Zur Ueberführung des Methylamido-Aethylphenylketons ' in das entsprechende Carbinol wurden 4,5 $\mathrm{g}$ des reinen, aus dem Platin-, bzw. Golddoppelsalz erhaltenen Hydrochlorids in etwa $100 \mathrm{ccm}$ Wasser gelöst, diese Lösung auf $0^{\circ}$ abgekühlt und in 
dieselbe, unter fortwährendem Umrühren, in Zwischenräumen von 10 Minuten, je $5 \mathrm{~g}$ Natriumamalgam von $5 \%$ und $5 \mathrm{~g}$ verdünnter Salzsäure (aus $43,5 \mathrm{~g}$ Salzsäure von $25 \%$ und $26,5 \mathrm{~g}$ Wasser bereitet) eingetragen. Im ganzen gelangten $40 \mathrm{~g}$ Natriumamalgam zur Anwendung. Die Temperatur überstieg hierbei $5^{0}$ nicht.

Nach Beendigung der Wasserstoffentwickelung wurde die saure Flüssigkeit zur Entfernung des sekundär gebildeten Propiophenons, zunächst wiederholt mit Aether ausgeschüttelt, dann mit Natronlauge neutralisiert, hierauf zur Trockne verdunstet und der Rückstand im Exsikkator noch vollständig ausgetrocknet. Diese Salzmasse wurde dann mit absolutem Alkohol extrahiert, der Auszug auf ein kleines Volum eingeengt und noch heiß mit Aceton versetzt. Beim Erkalten erfolgte eine Ausscheidung von Methylaminhydrochlorid in Gestalt von durchsichtigen, bei $2024^{\circ}$ schmelzenden Blättchen. Die hiervon ahgesogene Mutterlauge wurde verdunstet, der Rückstand in Wasser gelöst und die Lösung zur Darstellung eines Gold- und Platindoppelsalzes verwendet.

Das Goldd op pelsalz, welches in Wasser sehr schwer löslich war, schied sich zunächst ölig aus, jedoch nahm dasselbe nach Verlauf von einigen Tagen krystallinische Beschaffenheit an. Dasselbe erweichte bei $120^{\circ}$ und schmolz bei $126^{\circ}$.

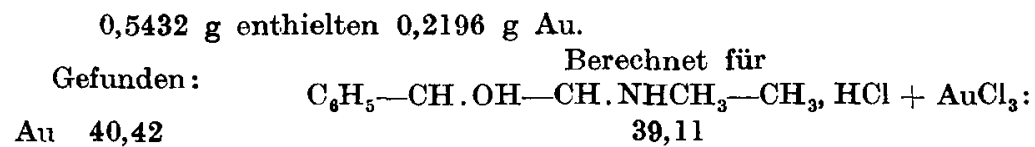

Der etwas zu hohe Goldgehalt ist durch eine geringe Beimengung von metallischem Gold bedingt.

Das P la t in d o p pels a I z ist wesentlich leichter in Wasser löslich als das entsprechende Golddoppelsalz. Dasselbe schied sich neben blätterigem Methylaminplatinchlorid zunächst auch nur in öligen Tröpfchen aus, die jedoch bald in rotgefärbte, warzenförmig gruppierte, bei $190-191^{\circ}$ schmelzende Krystalle übergingen. Dieselben konnten durch Abschlämmen und Auslesen isoliert werden.

$0,2679 \mathrm{~g}$ enthielten $0,0708 \mathrm{~g} \mathrm{Pt}$.

$$
\begin{array}{cc}
\text { Gefunden: } & \begin{array}{c}
\text { Berechnet für } \\
\text { Pt 26,43 }
\end{array} \quad\left(\mathrm{C}_{6} \mathrm{H}_{5}-\mathrm{CH} . \mathrm{OH}-\mathrm{CH} . \mathrm{NHCH}_{3}-\mathrm{CH}_{3}, \mathrm{HCl}\right)_{2} \mathrm{PtCl}_{4} \text { : }
\end{array}
$$

Das Hydrochlorid, welches aus diesen Doppelsalzen dargestellt wurde, war in Wasser und in Alkohol sehr leicht löslich, unlöslich in Aceton und in Aether. Beim Ueberschichten der 
konzentrierten alkoholischen Lösung mit Aether schied es sich in feinen, lockeren Nadeln aus, die bei $180-181^{\circ}$ schmolzen.

$0,0308 \mathrm{~g}$ verbrauchten zur Ausfällung des Chlors $1,54 \mathrm{ccm}$ 1/10-N.-Silbernitratlösung.
Gefunden :
Cl 17,73
Berechnet für $\mathrm{C}_{10} \mathrm{H}_{15} \mathrm{ON}, \mathrm{HCl}$ :
17,59

Die f r e i e B a s e schied sich beim langsamen Verdunsten ihrer ätherischen Lösung in feinen, verfilzten, bei $114-115^{\circ}$ schmelzenden Nadeln aus, welche einen schwachen, angenehmen, an Pseudoephedrin erinnernden Geruch besaßen.

Abgesehen von dem fehlenden Drehungsvermögen, zeigt die isolierte Base und ihre Verbindungen große Aehnlichkeit mit dem Pse ud oe phedrin. Ob dieselbe jedoch als inaktives Pseudoephedrin anzusprechen ist, sollen erst die weiteren, mit größerem Material ausgeführten Untersuchungen lehren. Ueber letztere wird später berichtet werden.

Eine Base der Formel $\mathrm{C}_{6} \mathrm{H}_{5}-\mathrm{CH} . \mathrm{OH}-\mathrm{CH} . \mathrm{NHCN}_{3}-\mathrm{CH}_{3}$ ist

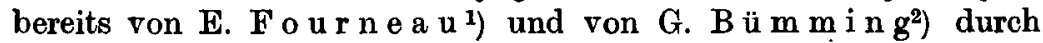
Einwirkung von Phenylpropylenjodhydrin auf Methylamin dargestellt worden. Fourneau fand den Schmelzpunkt des Hydrochlorids dieser Base bei $178^{\circ}$, B ü m m ing, nach dem Umkrystallisieren desselben aus Aceton, bei $190^{\circ}$. Dagegen soll die freie Base nach F o u r n e a u glänzende, bei $60^{\circ}$ schmelzende Prismen bilden, das Aurat ölige Beschaffenheit zeigen und das Platinat in Wasser sehr leicht löslich sein.

1) Journ. de Pharm. et de Chim. 1904, 481.

2) Inauguraldissertation, Marburg 1009. 